\title{
3D geostatistical inversion of induced polarization data and its application to coal seam fires
}

\author{
Abdellahi Soueid Ahmed ${ }^{1}$, André Revil ${ }^{1}$, Abdel Jardani ${ }^{2}$, and Rujun Chen ${ }^{2}$
}

\begin{abstract}
We applied the principal component geostatistical approach (PCGA) to the inversion of time-domain induced polarization data in terms of resistivity and chargeability distributions. The PCGA presents two major advantages over standard methods: (1) It avoids the storage of the usually large covariance matrix, which contains the geostatistical information, by factorizing it in a product of low-rank matrices. (2) It does not assemble the Jacobian matrix per se. We determine the robustness of this approach with three examples. We first reconstruct the electrical conductivity and chargeability fields of two synthetic models generated using the geostatistical software Stanford Geostatistical Modeling Software. The PCGA approach performs better than the Tikhonov-based regularization approach when the true fields are very heterogeneous and the amount of data is limited. The third example is devoted to a field study over the former Lewis coal mine
\end{abstract}

in Colorado (USA). We perform a 3D localization of the burning front of this coal seam fire by applying our geostatistical inverse methodology to a time-domain induced-polarization data set. In this case, the horizontal components of the semivariogram are determined from a self-potential map and the correlation length scale for the vertical component is determined from the known thickness of the coal bed. The tomogram presents a high normalized chargeability associated with the burning front. We evaluate the high normalized chargeability of the burning front in terms of the physical mechanism associated with the cation exchange capacity of the coal and the effect of temperature. This demonstrates the potential of the geostatistical inversion and its suitability for inverting geophysical data, especially when the data density is sparse. In the case of coal seam fires, we determine the suitability of the induced polarization method to localize the burning front and the effect of temperature on the normalized chargeability.

\section{INTRODUCTION}

Galvanometric induced polarization is a geophysical method used to image the ability of rocks to reversibly store electrical charges at low frequencies $(<1 \mathrm{kHz})$. This property is characterized by a material property called chargeability (between 0 for nonpolarizable media and 1 for perfectly polarizable media; see Seigel, 1949, 1959). Induced polarization can also be obtained through inductionbased electromagnetic methods such as time-domain electromagnetics (TDEM) (see Marchant et al., 2013, 2014; Macnae, 2015, 2016). Although induced-polarization effects have been known for a long time (Schlumberger, 1920), the method started to be extensively used only 40 years ago, mostly for mineral exploration (Van Voorhis et al., 1973; Pelton et al., 1978; Oldenburg et al., 1997). In fact, compared with other geophysical methods, induced polarization is particularly suitable for detecting disseminated ores due to their strong polarizations (Wong, 1979; Gurin et al., 2013; Marchant et al., 2013, 2014; Revil et al., 2017b, 2017c).

Nowadays, the range of induced polarization applications has remarkably broadened and covers, for instance, the tracking of contaminants plumes (Barker, 1990; Kemna et al., 2004; Sogade et al., 2006), landfill mapping (Leroux et al., 2010; Dahlin and Leroux, 2012; Gazoty et al., 2012), oil and gas exploration (Spies, 1983; Carlson and Zonge, 1996), monitoring of damage in porous rocks (Cosenza et al., 2016), and permeability characterization (Attwa and Günther, 2013; Revil et al., 2015b; Joseph et al., 2016) just to cite

Manuscript received by the Editor 17 April 2017; revised manuscript received 16 December 2017; published ahead of production 08 January 2018; published online 12 March 2018.

${ }^{1}$ Université Grenoble Alpes, CNRS, IRD, IFSTTAR, ISTerre, Grenoble, France and Université Savoie Mont Blanc, ISTerre, Chambéry, France. E-mail: abdellahi.soueid-ahmed@univ-smb.fr; andre.revil@univ-smb.fr.

${ }^{2}$ Université de Rouen, M2C, UMR 6143, CNRS, Morphodynamique Continentale et Côtière, Mont Saint Aignan, France. E-mail: abderrahim.jardani@ univ-rouen.fr.

(C) 2018 Society of Exploration Geophysicists. All rights reserved. 
a few examples. This is a consequence of the huge progress that has been made in terms of induced-polarization instrumentation (Kingman et al., 2007; Zimmermann et al., 2008; Xi et al., 2014) and processing as well as the inversion of the full waveform data (Olsson et al., 2016). In addition, our understanding of the background physics explaining induced-polarization phenomena has improved thanks to the recent development of petrophysical models such as the dynamic Stern layer model for porous media with no metallic particles (Rosen and Saville, 1991; Rosen et al., 1993; Revil and Florsch, 2010; Revil, 2012, 2013) and models based on redox phenomena and boundary polarization effects for dispersed metallic particles in porous media (Wong, 1979; Revil et al., 2015b, 2017b, 2017c).

However, traditional electrical induced-polarization inversion approaches (Loke and Barker, 1995, 1996; Binley and Kemna, 2005; Karaoulis et al., 2011) remain geostatistically deficient. Geologic formations are either not randomly built structures or are smooth. Therefore, classic regularization approaches may then omit important information on the spatial heterogeneity of the subsurface. Despite being widely used in the realm of hydrogeology, geostatistically based inversion approaches are still underused in geophysics. For instance, Maurer and Holliger (1998)use some statistical properties to build model covariance functions for constraining inverse problems. Linde et al. (2006) improve the characterization of a sandstone aquifer in the UK, by jointly inverting electrical resistivity tomography (ERT) and ground-penetrating radar data using stochastic-based regularization operators for geostatistical models. Yeh et al. (2002) developed a geostatistical approach based on a sequential linear estimator for ERT, whereas Johnson et al. (2012) use semivariogram constraints to carry out geostatistical inversion for electrical resistivity tomography. That said, the aforementioned algorithms were not based on the quasilinear geostatistical approach nor were they designed for reducing the dimensionality of large-scale problems. In the same vein, we propose using a geostatistical inverse scheme based on a method called the quasilinear geostatistical approach (QLGA), to invert 3D time-domain induced-polarization data.

The QLGA method, introduced by Kitanidis (1995), uses geostatistical information as prior information and then updates the parameter estimates using a linearization of the forward operator response. QLGA has been mostly developed and used in hydraulic tomography (HT) for which hydraulic conductivity and specific storage are imaged using pumping tests (e.g., Cirpka and Kitanidis, 2000; Pollock and Cirpka, 2012; Cardiff et al., 2012, 2013; Soueid Ahmed et al., 2014, 2015, 2016; Bakhos et al., 2014). Recently, a large-scale variant of QGLA, called the principal component geostatistical approach (PCGA), has been introduced by Kitanidis and Lee (2014) (see also Lee and Kitanidis, 2014). Kitanidis and Lee (2015) study in an unpublished work the computational benefits of using PCGA for ERT. With respect to computational efficiency, PCGA has the advantage of being matrix free; this means that it does not implicitly evaluate the Jacobian matrix, it only computes its products with other matrices or vectors. Although this is not specific to the PCGA itself (other methods such as nonlinear conjugate gradients [NLCG] also do not require evaluation of the Jacobian matrix), this significantly reduces the computational burden. In fact, in classical deterministic algorithms, computing the Jacobian matrix is the computationally heaviest step in the inversion process. Another major advantage of PCGA over conventional QLGA is that it decomposes the prior covariance matrix (which contains the prior geostatistical information) into a product of low-rank matrices. In other words, the large prior covariance matrix is replaced by a truncated matrix, which still contains the major information about the medium but is significantly smaller than the full initial matrix. This leads to consequent memory savings that will be discussed below.

The properties of the variogram (sill and correlation lengths) used for computing the covariance matrix must be known. Quantifying the uncertainties related to the geostatistical model is out of the scope of the current work. That said, there are several published studies exploring this issue. Essentially, these properties can be estimated from our prior knowledge of the subsurface structures, for instance, using geologic cross sections or estimated by fitting an experimental variogram to a theoretical one. Another approach consists of considering these properties as unknowns and estimating them during the inversion process (Kitanidis, 1995; Cardiff et al., 2012). The latter method is computationally very heavy and in many cases infeasible in 3D large-scale applications. However, some efforts have been recently made to accelerate the computation of uncertainty quantification in geostatistical inverse problems (e.g., Saibaba, 2013; Saibaba and Kitanidis, 2015). In the present work, we estimate the variogram properties using experimental variograms and geologic information as well.

The main goal of the present work is to develop a robust geostatistical inversion approach for time-domain induced-polarization data and to apply it to coal seam fires. We seek to reconstruct the 3D electrical resistivity and chargeability fields by inverting the apparent resistivities and apparent chargeabilities data usually collected at the ground surface and/or in boreholes. The inversion is carried out using the PCGA framework described above. Three case studies are examined to illustrate the effectiveness of our methodology. The first case corresponds to a moderately heterogeneous 3D synthetic test in which the true electrical resistivity and chargeability distributions are inverted and compared with the true fields. We also perform the inversion of the same fields using first-order derivative smoothing operators as constraints. In the second case, we show on a more heterogeneous synthetic model that the geostatistical inversion approach is more efficient than the classical Tikhonov-based regularization approach when the data density is limited. The third case considers field data collected during a 3D induced polarization field survey conducted over the former Lewis coal seam mine in Colorado (USA). The coal seam is still burning until today, and our goal is to perform a 3D inversion of the resistivity and chargeability data to localize the burning front and to understand the underlying mechanism of the anomalies.

\section{THEORY}

The 3D electrical potential field generated during a current injection in a heterogeneous isotropic medium is described by a Poisson's equation as

$$
\nabla \cdot\left(\sigma_{\infty} \nabla \Psi\right)+I \delta\left(x-x_{0}\right) \delta\left(y-y_{0}\right) \delta\left(z-z_{0}\right)=0,
$$

where $\sigma_{\infty}\left(\right.$ in $\left.\mathrm{Sm}^{-1}\right)$ denotes the instantaneous electrical conductivity field (see Figure 1a); $\Psi$ (in V) is the electrical potential generated by the injection of the current $I$ (in A); $\delta$ is the Dirac distribution; $x, y$, and $z$ represent the space locations; and $x_{0}, y_{0}$, and $z_{0}$ are the spatial coordinates of the current sources locations. The instantaneous conductivity is the conductivity of the material when induction effects have vanished but, still, all the charge carriers are mobile. This is by opposition to the steady-state conductivity $\sigma_{0}=(1-\eta) \sigma_{\infty}$ where $\eta$ 
denotes the dimensionless chargeability. This reduction $\left(\sigma_{0} \leq \sigma_{\infty}\right)$ occurs because the polarization of the material implies that some charge carriers are blocked at low frequencies and do not participate anymore to the conduction process.

Equation 1 is subject to the following boundary conditions:

$$
\begin{gathered}
\Psi=\alpha \quad \text { on } \Gamma_{1}, \\
\sigma_{\infty} \nabla \Psi \cdot \mathbf{n}=\beta \quad \text { on } \Gamma_{2},
\end{gathered}
$$

with $\Gamma_{1} \cup \Gamma_{2}=\partial \Omega$ where $\partial \Omega$ denotes the simulation domain boundaries, $\mathbf{n}$ denotes the outward unit vector normal to $\Gamma_{2}$. In the case of $\alpha=0$ and $\beta=0$, equations 2 and 3 correspond to homogeneous Dirichlet and homogeneous Neumann boundary conditions, respectively. Far from the bipole of current injection/retrieval (performed on electrodes A and B), a zero potential boundary condition is applied at infinity and insulating boundary conditions are applied at the ground surface. Once equation 1 is solved, one can compute the apparent resistivity through

$$
\rho_{a}=K \frac{\Delta \Psi}{I}
$$

where $K$ is the geometric factor accounting for the position of the electrodes, the topography, and the boundary conditions of the problem, $\Delta \Psi$ is the potential difference between two measuring (voltage) electrodes $M$ and $N$, and $I$ denotes the current injected in $A$ and retrieved at position $B$. Furthermore, equation 1 can be seen as a nonlinear mapping operator $F($.$) associating the electrical$ potential $\Psi_{\sigma_{\infty}}$ to the instantaneous conductivity field $\sigma_{\infty}$ :

$$
\Psi_{\sigma_{\infty}}=F\left(\sigma_{\infty}\right)
$$

This potential $\Psi_{\sigma_{\infty}}$ is instantaneously recorded when the current injection is turned on.

We come back now to the nature of the polarization. Porous materials are characterized by their ability to reversibly store electrical charges. This charge storage is associated with the polarization of the electrical double layer coating the mineral grains and the adjacent pore water (Vinegar and Waxman, 1984; Revil et al., 2015c). For disseminated metallic grains, the polarization can be associated with the accumulation of the charge carriers at the boundary of the grains (Wong, 1979; Revil et al., 2015a, 2015c). This ability is characterized by a material property called chargeability $\eta$ (with $0 \leq \eta \leq 1)$ and the existence of a phase lag between the current and the phase in frequency-domain induced polarization. To forwardmodel the chargeability, we follow an idea originally developed by Seigel (1959) and later taken up by various authors (Labrecque, 1991; Oldenburg and $\mathrm{Li}, 1994)$. The chargeability is taken into account by perturbing the electrical conductivity field during a current injection. In terms of numerical modeling, this means that the forward modeling of the time-domain effects is carried out just by solving two electrical resistivity direct current forward problems, one with the effective electrical conductivity distribution and the other with the same distribution but perturbed with the chargeability distribution. Indeed, considering that the ground is chargeable, we introduce chargeability effects by solving equation 1 using $\sigma_{0}=\sigma_{\infty}(1-\eta)$ as the input conductivity model, $\eta$ being the intrinsic chargeability, which is unitless but generally expressed in $\mathrm{mV} / \mathrm{V}$. The difference between the

instantaneous conductivity and the DC conductivity is explained in Figure 1 for an insulating grain coated by an electrical double layer.

The existence of the polarization allows for recording a voltage distribution defined as

$$
\Psi_{\sigma_{0}}=F\left(\sigma_{0}\right)
$$

This voltage corresponds to the voltage measured between $\mathrm{M}$ and $\mathrm{N}$ after a long current injection. Similarly, an apparent chargeability can be computed as (e.g., Li and Oldenburg, 2003)

$$
\eta_{a}=\frac{\Psi_{\sigma_{\infty}}-\Psi_{\sigma_{0}}}{\Psi_{\sigma_{\infty}}}=\frac{F\left(\sigma_{\infty}\right)-F\left(\sigma_{0}\right)}{F\left(\sigma_{\infty}\right)}
$$

Therefore, modeling the time-domain induced polarization requires solving equation 1 twice with different electrical resistivity distributions (Seigel, 1959). It is important to mention here that equation 7 may lead to numerical instabilities if $F\left(\sigma_{\infty}\right) \approx F\left(\sigma_{0}\right)$. Therefore, it is crucial to have a highly accurate forward response to minimize these errors as much as possible.

In time-domain induced-polarization surveys, we generally compute the partial chargeability expressed in milliseconds and defined as

$$
\eta_{t_{j}, t_{j+1}}=\frac{1}{\psi_{\sigma}} \int_{t_{j}}^{t_{j+1}} \Psi(t) d t
$$

where $\eta_{t_{j}, t_{j+1}}$ denotes the partial chargeability measured during the time window $\left[t_{j}, t_{j+1}\right]$ and $\Psi(t)$ is the decaying voltage, measured just after the current is shut off.

a)
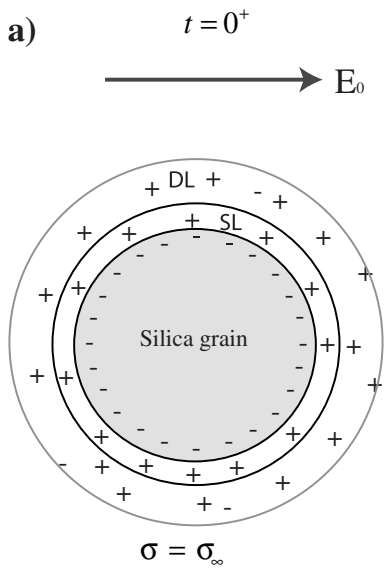

Figure 1. Difference between the instantaneous conductivity $\sigma_{\infty}$ and the DC conductivity $\sigma_{0}$ for a silica grain coated by an electrical double layer (a) Right after the application of the primary electrical field $\mathbf{E}_{0}$, all the ionic charge carriers are mobile through the process of electromigration. The instantaneous conductivity is $\sigma_{\infty}$. (b) If the primary electrical field is applied for a long time (DC condition), the conductivity is $\sigma_{0}=\sigma_{\infty}(1-M)$, where $M$ stands for the chargeability of the material (dimensionless). Some of the charge carriers (those of the Stern layer, i.e., the inner part of the double layer) are now blocked at the edge of the grain in the direction of the applied electrical field. As a result, the conductivity of the material is reduced. This conductivity reduction also characterizes the polarization of the grain. 
Likewise, partial chargeability can be expressed in $\mathrm{mV} / \mathrm{V}$ as (Florsch et al., 2011)

$$
\eta_{t_{i}, t_{i+1}}=\frac{1}{\psi_{\sigma}\left(t_{j+1}-t_{j}\right)} \int_{t_{j}}^{t_{j+1}} \Psi(t) d t .
$$

Partial chargeability can be related to the intrinsic chargeability $\eta$ through the approximation relation (e.g., Florsch et al., 2011):

$$
\eta_{t_{i}, t_{i+1}} \approx \eta\left(t_{j+1}-t_{j}\right)
$$

Note that this approximation is in principle strictly valid only under the condition that $t_{j}, t_{j+1} \ll \tau_{0}$, where $\tau_{0}(\mathrm{~s})$ is the time constant $\left(\tau_{0}\right.$ basically refers to the time needed for a polarized material to go back to its initial equilibrium state).

\section{GEOSTATISTICAL INVERSION APPROACH}

In this section, we present the inversion methodology used to invert the induced-polarization data with the goal to retrieve the electrical resistivity and chargeability spatial distributions. As a matter of fact, when dealing with time-domain induced polarization, two strategies can be used for inverting such distributions: (1) We first estimate the electrical resistivity, and then we use the resistivity tomogram for the inversion of the chargeability. (2) We perform simultaneous inversion of both parameters iteration by iteration. The first strategy is easier to compute and provides higher accuracy if the electrical resistivity is well estimated. In the current work, we opt for the first strategy using the PCGA method (Kitanidis and Lee, 2014; Lee and Kitanidis, 2014).

As stated before, the major difficulty in solving inverse problems lies in the nonuniqueness of the solution. Different strategies can be adopted to address this issue. The majority of inverse algorithms used in geophysics inversion use regularization via smoothing constraints to obtain the optimal estimated parameters model. However, this strategy leads to oversmoothed parameter models that, although they fit the measured data, do not necessarily preserve the features and magnitudes of the true model. An interesting alternative is the use of geostatistical-based inversion approaches that attack the issue of the nonuniqueness from a different perspective by using the geostatistical properties of the medium as constraints for guiding the inversion. The degree of heterogeneity of a medium can be described by a variogram. The main advantage of geostatistical inversion methods over traditional inversion ones is the incorporation of such "geostatistical behavior" in the inverse framework by casting this geostatistical information into a prior spatial covariance matrix. Giving this valuable a priori information helps obtaining more geologically meaningful estimated parameters fields. Because in geophysical applications the dimensionality of the inverse problem usually needs to be high to obtain satisfactory resolution of the inverted models, we opt for a large-scale geostatistical method (the PCGA).

As mentioned above, the PCGA has several advantages. It does not necessitate the evaluation of the Jacobian matrix. Instead, it only computes its products. As a direct consequence, this significantly reduces the computational time when we are dealing with large datasets and high number of unknowns resulting in very large Jacobian matrices that are heavy to assemble and store. Moreover, the PCGA reduces the dimensionality of our inverse problem by factorizing the prior covariance matrix in a product of low-rank matrices. In addition, the PCGA offers the possibility of computing uncertainty maps of the estimated parameters in a computationally economic way. All the aforementioned points make the PCGA an appealing inversion approach especially for geophysical applications. In conventional QGLA, we seek to solve the following minimization problem whose solution corresponds to the best inverted model:

$$
\min _{\mathbf{p}} L(\mathbf{p})
$$

with

$$
\begin{aligned}
L(\mathbf{p})= & \frac{1}{2}(\mathbf{d}-F(\mathbf{p}))^{T} \mathbf{V}^{-1}(\mathbf{d}-F(\mathbf{p})) \\
& +\frac{1}{2}(\mathbf{p}-\mathbf{X} \beta)^{T} \mathbf{Q}^{-1}(\mathbf{p}-\mathbf{X} \beta)
\end{aligned}
$$

where $L$ denotes the objective function; $\mathbf{p}$ is the $m \times 1$ parameter vector, whether the electrical resistivity $\rho$ or the chargeability $\eta$; $\mathbf{d}$ is the $n \times 1$ observed data vector, whether apparent electrical conductivities or apparent chargeabilities; $F($.$) is the forward operator; \mathbf{V}$ is the $n \times n$ data covariance matrix; $\mathbf{X}$ is an $m \times n_{p}$ matrix, where $n_{p}$ is the number of physical parameters to be estimated; $\beta$ is the drift coefficient; and $\mathbf{Q}$ denotes the $m \times m$ prior spatial covariance matrix that is represented by a variogram. As we can see, $L$ is composed of two terms: The first one handles the data misfit, and the second one assures, through imposing constraints, that the inverted model reflects the heterogeneities of the field. One of the major ideas that underlies the PCGA is the factorization of the covariance matrix $\mathbf{Q}$ in a product of low-rank matrices. For instance, using an eigendecomposition factorization yields:

$$
\begin{gathered}
\mathbf{Q} \approx \mathbf{Q}_{K}=\mathbf{P}_{K} \boldsymbol{\Lambda}_{K} \mathbf{P}_{K}^{T}=\sum_{i=1}^{K} \mathbf{u}_{i} \mathbf{u}_{i}^{T}, \\
\mathbf{u}_{i}=\sqrt{\left|\lambda_{i}\right|} \mathbf{P}_{i},
\end{gathered}
$$

where $K$ is the truncation order, $\mathbf{Q}_{K}$ is the $K \times K$ truncated covariance matrix, $\boldsymbol{\Lambda}_{K}$ is the $K \times K$ diagonal matrix of the first $K$ largest eigenvalues of $\mathbf{Q}, \mathbf{P}_{K}$ is the $K \times m$ matrix of the corresponding eigenvectors $\mathbf{u}_{i}$, and $\lambda_{i}$ is the $i$ th eigenvalue of $\mathbf{Q}_{K}$. Therefore, instead of working with the large matrix $\mathbf{Q}$, we replace it by its low-rank approximation, which leads to significant storage savings because $K \ll m$. In light of this, it is important to realize that the leading information in the matrix $\mathbf{Q}$ is covered by the largest eigenvalues; therefore, we can obtain very satisfactory tomography results by replacing $\mathbf{Q}$ with $\mathbf{Q}_{K}$, provided that we choose a reasonable value of $K$. The choice of an optimal truncation order $K$ is a crucial point for having good tomography results. Examining the spectrum of $\mathbf{Q}$ helps determining the optimal truncation order $K$. In fact, the relative error of the low-rank approximation is given by

$$
\varepsilon=\frac{\left\|\mathbf{Q}-\mathbf{Q}_{K}\right\|}{\|\mathbf{Q}\|}=\frac{\lambda_{K+1}}{\lambda_{1}},
$$

where $\lambda_{K+1}$ is the $K+1$ th eigenvalue of $\mathbf{Q}$ and $\lambda_{1}$ is the largest eigenvalue of $\mathbf{Q}$. Therefore, one can choose $K$ so that $\varepsilon$ is sufficiently small. In our case, the eigenvalues decay is very fast so moderate values of 
$K$ are sufficient for capturing the leading information of the eigenspace of $\mathbf{Q}$.

It follows that a more computationally appealing version of the objective function $L$ (equation 12) can be written as (Kitanidis and Lee, 2014)

$$
L(\mathbf{p})=\frac{1}{2}(\mathbf{d}-F(\mathbf{p}))^{T} \mathbf{V}^{-1}(\mathbf{d}-F(\mathbf{p}))+\frac{1}{2} \mathbf{p}^{T} \mathbf{P}_{K} \boldsymbol{\Lambda}_{K}^{-1} \mathbf{P}_{K}^{T} \mathbf{p} .
$$

The solution of the minimization problem 11 can be found using an iterative process in which the current optimal parameter model in each iteration is given by (Kitanidis and Lee, 2014)

$$
\mathbf{p}_{i+1}=\mathbf{A}\left(\mathbf{d}-F\left(\mathbf{p}_{i}\right)+\mathbf{J}_{i} \mathbf{p}_{i}\right),
$$

where $\mathbf{p}_{i}$ is the current best model estimate at iteration $i, \mathbf{J}_{i}$ is the Jacobian matrix at iteration $i$, given by $\mathbf{J}_{i}=\partial F(\mathbf{p}) /\left.\partial \mathbf{p}\right|_{\mathbf{p}=\mathbf{p}_{i}}, \mathbf{A}$ is obtained by solving the following linear system:

$$
\left(\begin{array}{cc}
\mathbf{J}_{i} \mathbf{Q} \mathbf{J}_{i}^{T}+\mathbf{V} & \mathbf{J}_{i} \mathbf{X} \\
\left(\mathbf{J}_{i} \mathbf{X}\right)^{T} & 0
\end{array}\right)\left(\begin{array}{c}
\mathbf{A}^{T} \\
\mathbf{B}
\end{array}\right)=\left(\begin{array}{c}
\mathbf{J}_{i} \mathbf{Q} \\
\mathbf{X}^{T}
\end{array}\right)
$$

As stated before, a key point of the PCGA resides in the fact that the Jacobian matrix $\mathbf{J}$ is never computed; instead, its products are computed as

$$
\mathbf{J X}_{i} \approx \frac{1}{\delta}\left[F\left(\mathbf{p}+\delta \mathbf{X}_{i}\right)-F(\mathbf{p})\right]
$$

where $\mathbf{X}_{i}$ denotes the $i$ th column of $\mathbf{X}$ and $\delta$ is the finite-differences step. The choice of $\delta$ is important. It must not be too small, to avoid numerical instabilities, and it must not be too large, to maintain the high accuracy of the finite-differences approximation. In the present paper, we empirically choose $\delta=0.001$, yet other ways of choosing $\delta$ are discussed in Lee and Kitanidis (2014). The product JQ is computed by

$$
\mathbf{J Q} \approx \mathbf{J Q}_{K}
$$

$$
\mathbf{J Q} \approx \mathbf{J} \sum_{i=1}^{K} \mathbf{u}_{i} \mathbf{u}_{i}^{T}
$$

$$
\mathbf{J Q} \approx \sum_{i=1}^{K}\left(\mathbf{J} \mathbf{u}_{i}\right) \mathbf{u}_{i}^{T}
$$

$$
\mathbf{J Q} \approx \sum_{i=1}^{K} \boldsymbol{\varsigma}_{i} \mathbf{u}_{i}^{T}
$$

where

$$
\boldsymbol{\varsigma}_{i}=\mathbf{J u}_{i}=\frac{1}{\delta}\left[F\left(\mathbf{p}+\delta \mathbf{u}_{i}\right)-F(\mathbf{p})\right]
$$

The remaining term in equation 18 can be approximated as follows:

$$
\mathbf{J Q J} \mathbf{J}^{T} \approx \mathbf{J} \mathbf{Q}_{K} \mathbf{J}^{T}
$$

$$
\mathbf{J Q J} \mathbf{J}^{T} \approx \sum_{i=1}^{K}\left(\mathbf{J u}_{i}\right)\left(\mathbf{J} \mathbf{u}_{i}\right)^{T}
$$

Table 1. Summary of the synthetic case study and Lewis fire case study simulation parameters.

Property

Dimension

Simulation domain

Number of unknowns

Number of measurements

Number of iterations

Number of profiles

Number of electrodes

Synthetic case study

$3 \mathrm{D}$

$162 \mathrm{~m} \times 20 \mathrm{~m} \times 30 \mathrm{~m}$

48,000

620 for ERT, 620 for chargeability tomography 6 for PCGA, 8 for GN

5

32 per profile
Lewis fire case study

3D

$162 \mathrm{~m} \times 20 \mathrm{~m} \times 30 \mathrm{~m}$

48,000

629 for ERT, 629 for chargeability tomography 6 for PCGA, 8 for GN

5

32 per profile

Electrical resistivity variogram

$$
\begin{aligned}
& \gamma(h)=0.91\left(1-\exp \left(-\left(\frac{h}{9.61}\right)^{2}\right)\right) \\
& \gamma(h)=0.12\left(1-\exp \left(-\left(\frac{h}{15.47}\right)^{2}\right)\right)
\end{aligned}
$$$$
\gamma(h)=0.003\left(1-\exp \left(-\sqrt{\left(\frac{h_{x}}{12}\right)^{2}+\left(\frac{h_{y}}{50}\right)^{2}+\left(\frac{h_{z}}{5}\right)^{2}}\right)\right)
$$

Chargeability variogram

Truncation order

30 for test 1 and 100 for test 2 
a)

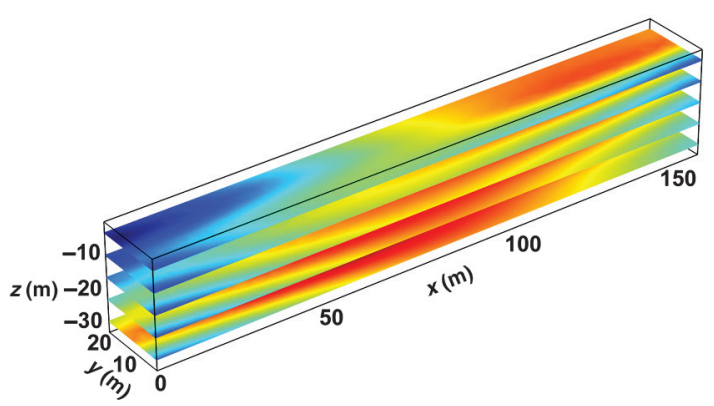

c)

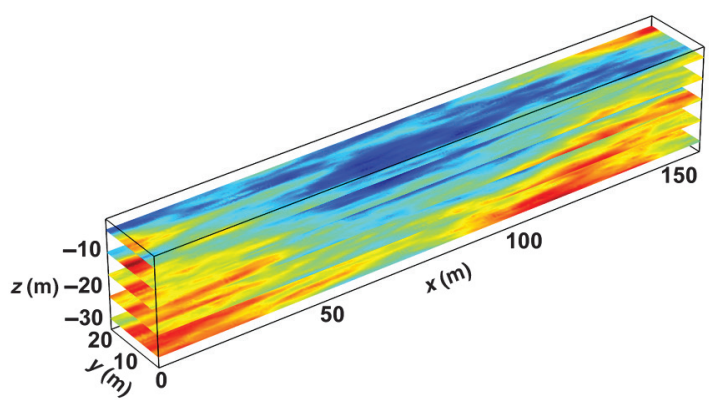

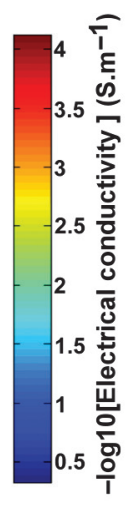

b)

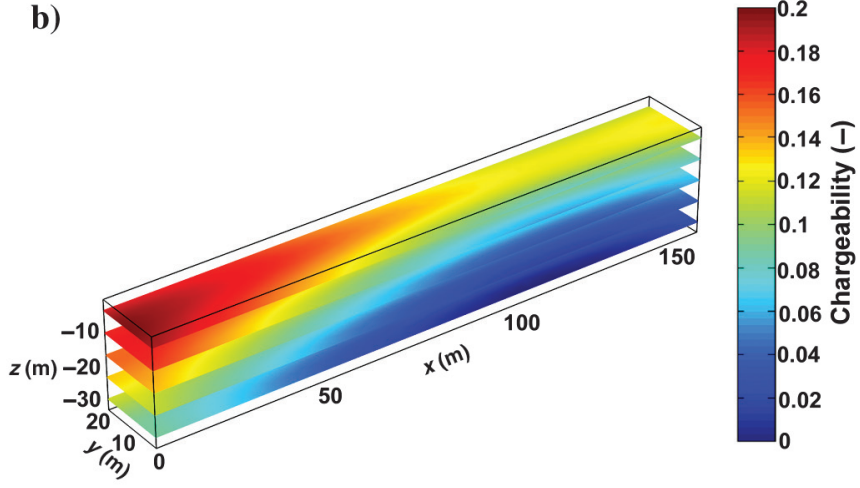

d)

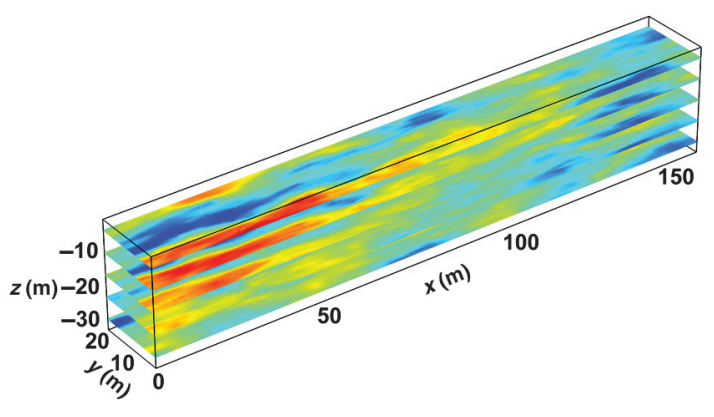

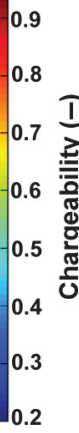

Figure 2. True synthetic distributions. (a) Synthetic electrical conductivity distribution for test 1 . (b) Synthetic intrinsic chargeability distribution for test 1. (c) Synthetic electrical conductivity distribution for test 2. (d) Synthetic intrinsic chargeability distribution for test 2. These fields were generated using the geostatistical software SGEMS. They are used as true parameter fields for the first case study. During the inversion process, these fields are assumed to be unknown and we estimate their spatial distributions using the PCGA and GN methods.
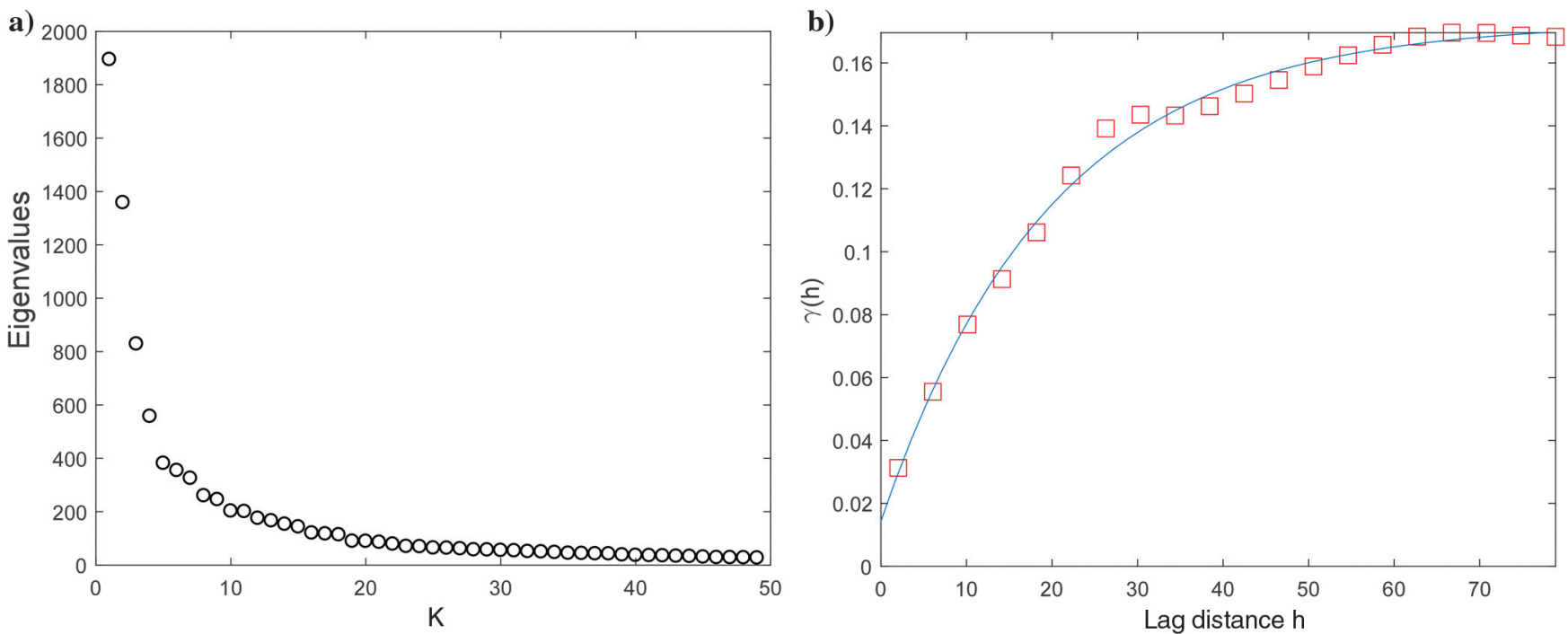

Figure 3. Covariance matrix and variograms used in test 2. (a) Leading eigenvalues of the covariance matrix used for the inversion of the electrical conductivity. (b) Experimental and theoretical variograms used for the electrical conductivity tomography. The experimental variogram is fitted to an exponential one, using a least-squares minimization. 


$$
\mathbf{J Q J}^{T} \approx \sum_{i=1}^{K} \boldsymbol{s}_{i} \boldsymbol{s}_{i}^{T}
$$

The matrix products from equations $19-22$ require $K+3$ forward runs per iteration, whereas a classical inversion approach will require $m+1$ forward runs if performed with the perturbation method and $n+1$ with the adjoint-state method. Therefore, the use of the PCGA indeed leads to significant computational savings. Once the objective function has decreased and the changes in its values became moderate through the iterative algorithm process, we consider that convergence has been reached and the iterative process can be stopped.

The algorithm of the PCGA is summarized below (Lee and Kitanidis, 2014):

a)
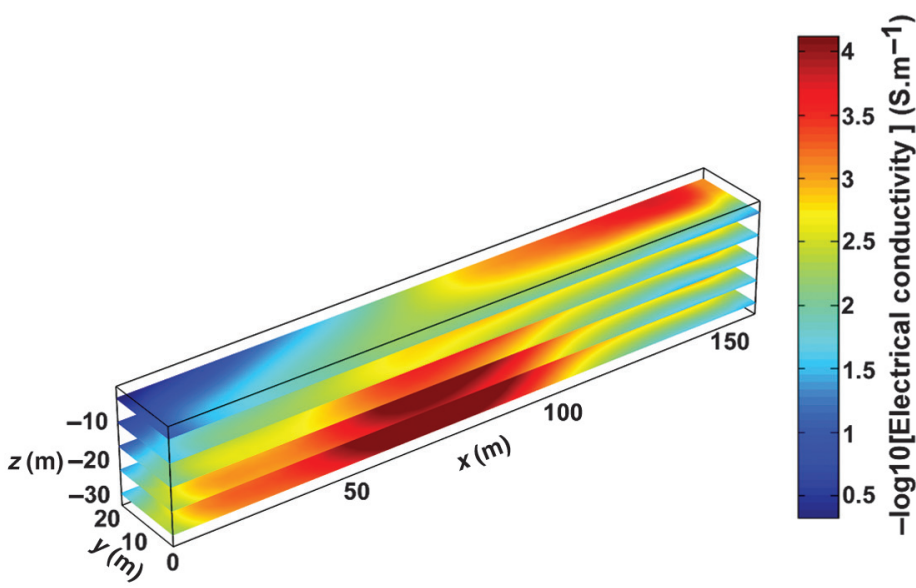

c)

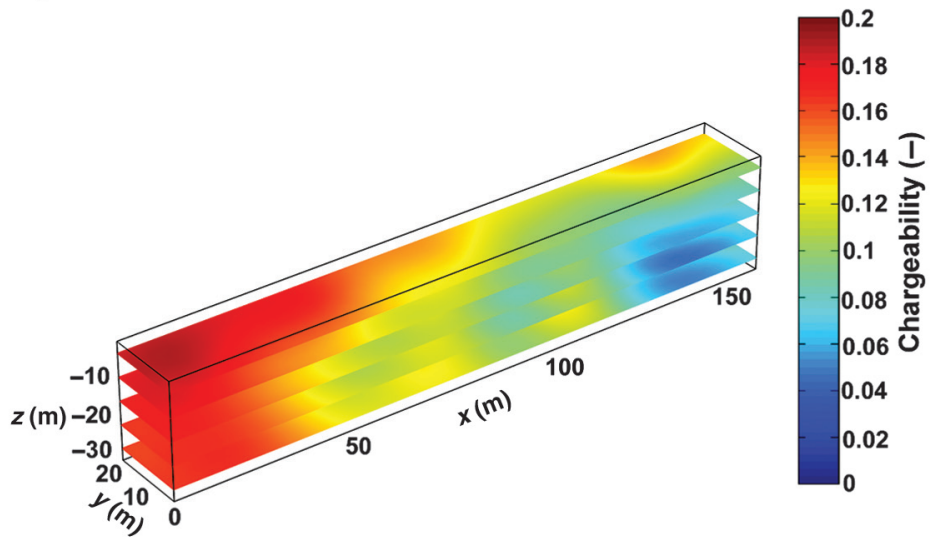

1. Compute the low-rank approximation $\mathbf{Q}$.

2. Compute the $H_{i}$ products: $\mathbf{J}_{i} \mathbf{Q}_{K}, \mathbf{J}_{i} \mathbf{X}_{i}, \mathbf{J}_{i} \mathbf{Q}_{K} \mathbf{J}_{i}^{T}$.

3. Solve the linear system

$$
\left(\begin{array}{cc}
\mathbf{J}_{i} \mathbf{Q} \mathbf{J}_{i}^{T}+\mathbf{V} & \mathbf{J}_{i} \mathbf{X} \\
\left(\mathbf{J}_{i} \mathbf{X}\right)^{T} & 0
\end{array}\right)\left(\begin{array}{c}
\mathbf{A}^{T} \\
\mathbf{B}
\end{array}\right)=\left(\begin{array}{c}
\mathbf{J}_{i} \mathbf{Q} \\
\mathbf{X}^{T}
\end{array}\right) .
$$

4. Update the solution $p_{i}$

$$
\mathbf{p}_{i+1}=\mathbf{A}\left(\mathbf{d}-F\left(\mathbf{p}_{i}\right)+\mathbf{J}_{i} \mathbf{p}_{i}\right) .
$$

5. Repeat steps 2 to 4 , until the convergence criteria are met.

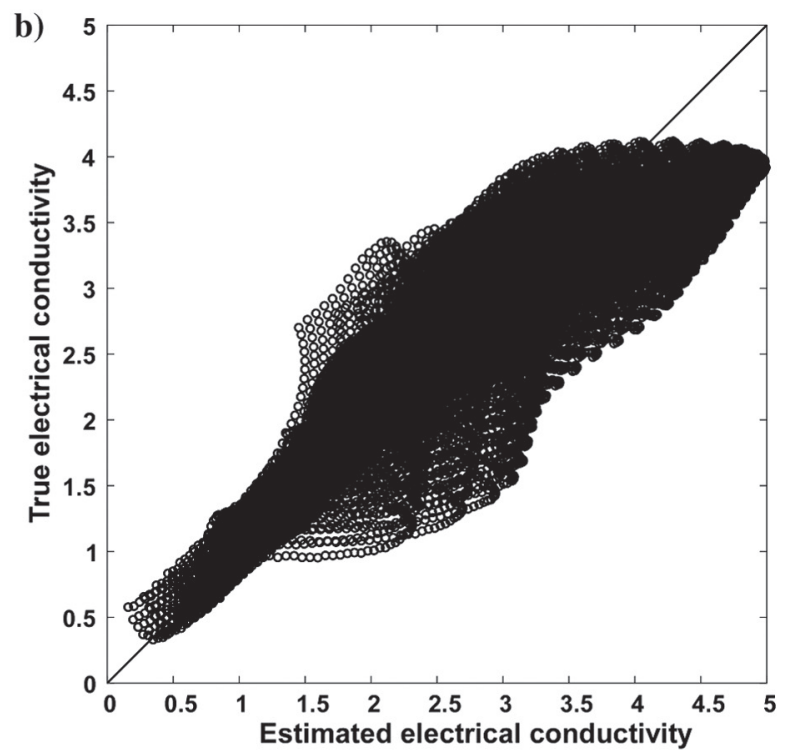

d)

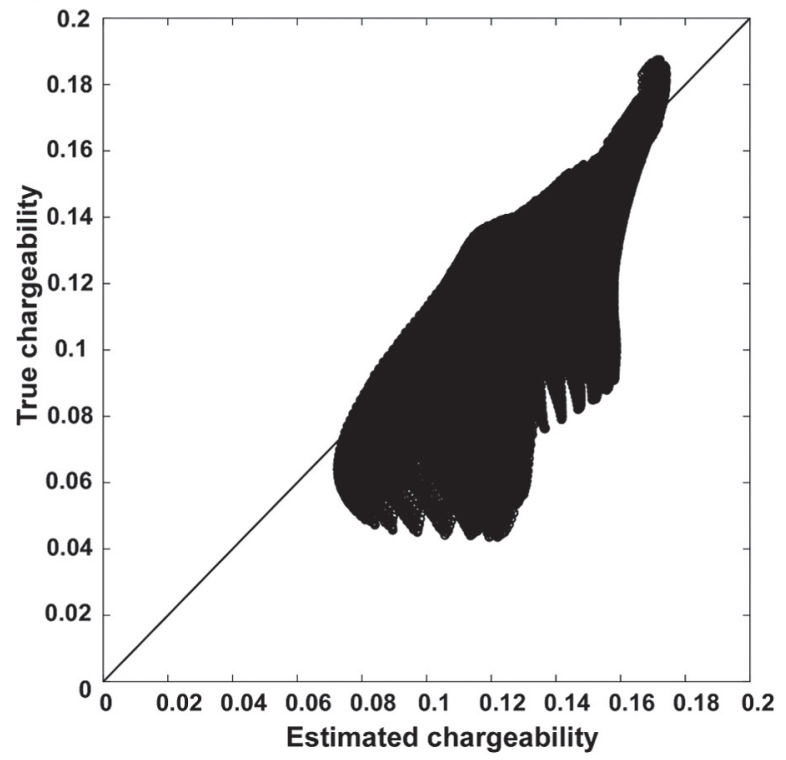

Figure 4. Tomography results of test 1 using the PCGA. (a) Estimated electrical conductivity field tomogram. (b) Scatterplot of the true electrical conductivity field against the estimated one. (c) Estimated chargeability field tomogram. (d) Scatterplot of the true chargeability field against the estimated one. 


\section{BENCHMARK TESTS}

We first benchmark our method using two synthetic cases. First, we generate an electrical conductivity field and a chargeability field. Then, we simulate the acquisition of a field survey by computing the apparent conductivities and chargeability data that would be measured on a given set of electrodes. These synthetic data are contaminated with noise. The last step is to invert them using the algorithm proposed above to determine the distribution of the conductivity and chargeability fields and compare them with the true fields (benchmark procedure). In addition, we invert the same noise-contaminated apparent conductivity and chargeability synthetic data using the traditional Gauss-Newton $(\mathrm{GN})$ method with a regularization done via first-order derivative smoothing.

We assign Neumann's boundary conditions (equation 3) at the ground surface to account for the insulating boundary condition at this interface. During the forward modeling, the region of interest is embedded into a much larger domain to account for the fact that, in reality, the measurements take place over a half-space, and homogeneous Dirichlet's boundary conditions (equation 2) are assigned to the remaining boundaries; therefore, they are far enough from the area of interest (the same will be done for the field case study described later).

Our synthetic target region covers a volume of $162 \mathrm{~m} \times 20 \mathrm{~m} \times 30 \mathrm{~m}$. The synthetic induced-polarization measurements are collected on five profiles. Each profile is characterized by 32 equally spaced electrodes. The electrodes lateral spacing is $1 \mathrm{~m}$, and the profiles are separated by $4 \mathrm{~m}$. We use a Wenner- $\alpha$ protocol resulting in 620 apparent conductivity and 620 apparent chargeability measurements. The simulation domain is discretized into 48,000 cells. We assign to each cell constant electrical conductivity and chargeability values, and we start the inversion of the parameters with homogeneous fields, corresponding to the geometric mean of the apparent conductivity and chargeability. Although some authors choose the apparent resistivity or chargeability pseudosections as initial models, our preference was launching the inversion with more neutral, blank homogeneous models corresponding to the geometric mean of the apparent parameter fields.

We used the geostatistical software SGEMS (Deutsch and Journel, 1992) to generate the synthetic electrical conductivity and chargeability fields of the two synthetic models (Figure 2). The

Table 2. Root-mean-square (rms) errors of the synthetic and Lewis fire case studies.

\begin{tabular}{lllllll}
\hline \multirow{2}{*}{ Parameter } & \multicolumn{2}{c}{ Test 1} & \multicolumn{2}{c}{ Test 2} & \multicolumn{2}{c}{ Lewis fire } \\
\cline { 2 - 7 } & GA & GN & GA & GN & GA & GN \\
\hline$\sigma_{a}$ & 0.02 & 0.011 & 0.012 & 0.08 & 0.149 & 0.146 \\
$\eta_{a}$ & 0.01 & 0.009 & 0.011 & 0.018 & 0.0142 & 0.42 \\
$\sigma$ & 0.40 & 0.24 & 0.20 & 0.27 & - & - \\
$\eta$ & 0.015 & 0.01 & 0.06 & 0.03 & - & -
\end{tabular}

The quantity $\eta_{a}$ denotes the apparent chargeability, $\sigma_{a}$ denotes the apparent electrical conductivity, $\sigma$ denotes the electrical resistivity (in ohm-m), and $\eta$ denotes the chargeability (comprised between 0 and 1, dimensionless). The rms is based on the square root of the summation of the residuals. In other words, for an observed value $z$, rms $=\left(1 / n_{z}\right)$ $\left(\sum_{i=1}^{n_{z}}\left(z_{i}-\hat{z}_{i}\right)^{2}\right)^{1 / 2}$, in which, $n_{z}$ denotes the number of elements in $z$ and $\hat{z}$ is the vector of predicted values of $z$. All the conductivity rms values are expressed in S.m ${ }^{-1}$. fields of the first test case are moderately heterogeneous, whereas those of the second one are more heterogeneous. All these fields were generated using exponential variograms whose properties are reported in Table 1. The properties of the variograms are considered to be unknown and are estimated using an experimental variogram that is fitted to a theoretical one (Figure $3 b$ ). The synthetic data have been contaminated with an artificial Gaussian random noise ( $2 \%$ of the mean of data).

The forward modeling defined by equations $1-3$ is realized using the finite-element software COMSOL Multiphysics 5.3. To get high accuracy of the forward calculations, the finite-element mesh is refined around the electrodes whereas a coarser mesh is used for the larger buffer domain. We have checked that the result of the forward modeling is independent of the mesh (the same will be done for the case study below). For all simulations, the eigenvalues decay was so fast that the leading information contained in the covariance matrices is given by the largest eigenvalues (see Figure 3a). Therefore, a small $K$ can be enough to obtain the tomograms of the inverted fields that have a satisfactory resolution. In our case, we used $K=25$ for the first synthetic case and $K=100$ for the second synthetic case. The relative errors $\lambda_{K+1} / \lambda_{1}$ associated to the electrical conductivity and chargeability covariance matrices, respectively, are 0.0046 and 0.0041 for the first synthetic case study and $3.1456 \times 10^{-4}$ and $6.7054 \times 10^{-4}$ for the second synthetic case study.

We start by discussing the results of the first synthetic test case. We first use the PCGA to invert the apparent conductivity data to image the 3D spatial heterogeneities of the electrical conductivity field. The inverted model is represented in Figure 4a. We can observe that the anomalies are well-recovered. The field root-meansquare (rms) error is very low (see Table 2): The plot of the true field against the estimated one clearly shows a linear trend (see Figure $4 \mathrm{~b})$, and the $R^{2}$ coefficients are very high $\left(R^{2}=0.802\right)$. The estimated chargeability field reflects in a satisfactory way the main heterogeneities of the true chargeability field. The shapes and magnitudes of the anomalies are reasonably retrieved as well. The rms exhibits a quite low uncertainty rate: 0.0153 , proving that the inversion approach successfully reconstructed the fields. For comparison purposes (see Figure 5), we show the results obtained using the traditional GN method.

We notice that the GN results are satisfactory as well, especially for the electrical conductivity field, which has roughly the same $R^{2}(0.79)$ as for the one reconstructed using the PCGA. The rms is even lower

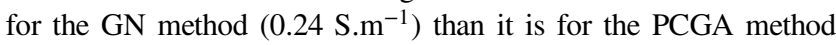
$\left(0.40 \mathrm{~S} . \mathrm{m}^{-1}\right)$. The chargeability field anomalies are, however, slightly better reconstructed using the PCGA (compare Figures $4 \mathrm{c}$ and $5 \mathrm{c}$ ). The rms values are almost the same for both methods: 0.0153 for PCGA and 0.0159 for GN. The $R^{2}$ are higher for the PCGA: 0.52 and 0.39 for GN. For this synthetic test, the PCGA and GN performed similarly in terms of the resolution of the inverted tomograms. Regarding the computational times, the PCGA performs better than GN.

We now consider the second synthetic test. We recall that this test has the same geometry and electrodes configuration as the first synthetic test. That said, the level of heterogeneity is higher to show which technique would be able to recover the parameter fields under the restriction of having a limited amount of data. Figure $6 \mathrm{a}$ and $6 \mathrm{c}$ shows the inverted electrical conductivity and chargeability fields obtained using the PCGA approach. The main features of these two fields (resistivity and chargeability) are well-recovered in terms 
of delineation and magnitude. The scatterplots of the true fields against the estimated ones follow a linear trend (Figure $6 \mathrm{~b}$ and 6d). The results obtained with the GN approach are shown in Figure 7. The inverted tomograms are oversmooth and do not properly recover the spatial features of the true fields. In addition, the scatterplots do not exhibit a linear trend. The $R^{2}$ values (see Table 3 ) are much greater for the PCGA approach. We underline that despite the low resolution of its tomograms, the GN still fairly reproduce the data (apparent conductivity: $\mathrm{rms}=0.08 \mathrm{~S} . \mathrm{m}^{-1}, R^{2}=0.85$; apparent chargeability: $\mathrm{rms}=0.018, R^{2}=0.95$ ). The obtained tomogram is, however, too smooth and displays a lower resolution than the one obtained with PCGA approach. The major features of the two fields are not well-recovered with the GN approach. This shows the aforementioned inherent difficulty in addressing the issue of nonuniqueness of the inverse problem and brings out the rationale for using a more robust inversion approach, such as PCGA, that gives estimated models that not only fit the observed data but also reflect some additional information regarding the level of heterogeneity of the subsurface.

The purpose of this synthetic test is not to question the efficiency of the widely used GN method. We just underline the fact that when we only have a limited number of measurements, introducing geostatistical constraints helps in guiding the inverse algorithm toward more physically meaningful models, by avoiding oversmoothing across the features. Undoubtedly, if we increase the number of induced-polarization measurements, the conventional GN method may provide better results. But again, this brings out the rationale for using geostatistical methods when we do not have enough a)
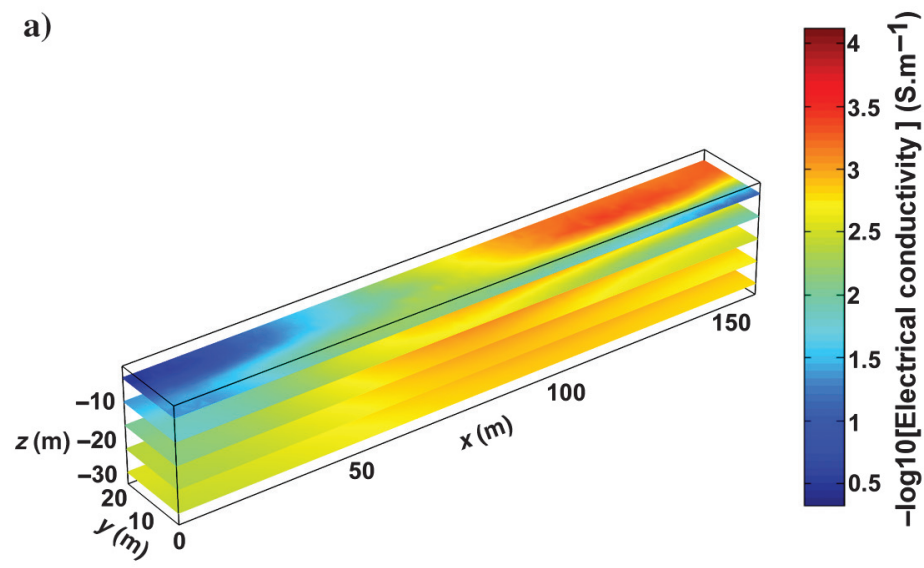

c)

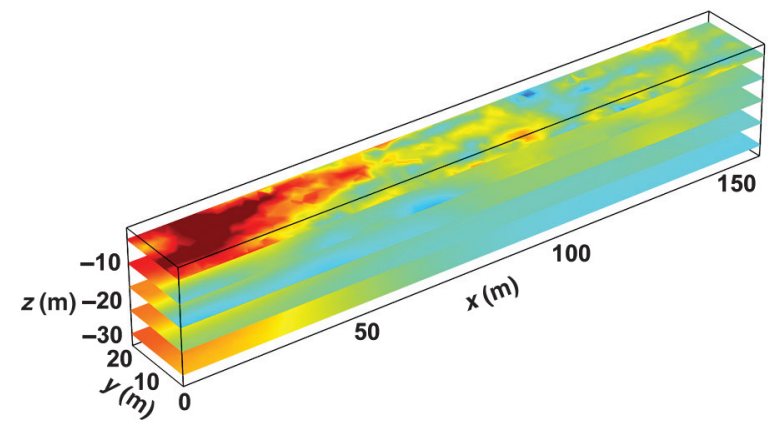

b)

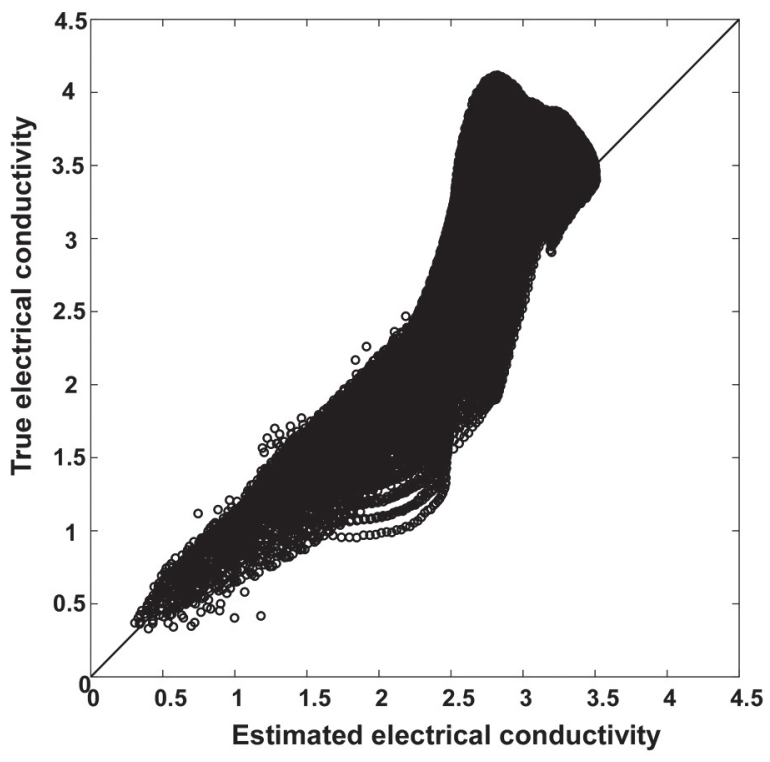

d)

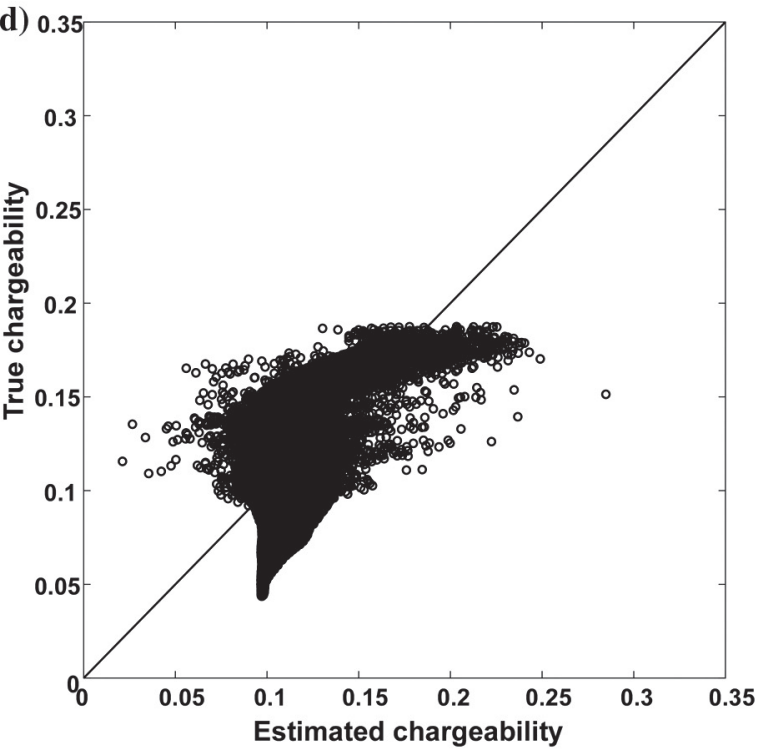

Figure 5. Tomography results of test 1 using the GN method. (a) Estimated electrical conductivity field tomogram. (b) Scatterplot of the true electrical conductivity field against the estimated one. (c) Estimated chargeability field tomogram. (d) Scatterplot of the true chargeability field against the estimated one. 
data to adequately constrain the inverse problem. From these two synthetic tests, we were also able to show the computational efficiency of PCGA over the conventional GN method. Despite that for the GN inversion we speeded up the computations of the Jacobian matrix using the adjoint method instead of the perturbation method, the PCGA computational times remain smaller than the GN ones (see Table 5). This better clarifies our claims about the computational efficiency of the PCGA approach.

\section{LABORATORY EXPERIMENT}

As in our field application, we will work on the induced polarization response of burning coal, we first perform a laboratory experiment to support the feasibility of the induced polarization method to delineate coal seam fire fronts. With this goal in mind, we perform a laboratory spectral induced-polarization experiment using coal powder (grain size of $\sim 1 \mathrm{~mm}$ ). The experiments are performed with the ZEL-SIP04-V02 impedance meter developed by Egon Zimmermann at the Central Laboratory for Electronics, ZEL, the Forschungszentrum Julich (see Zimmermann et al., 2007, 2008). The sample holder (see Revil and Skold, 2011) is put in a plastic bag immersed in a thermostatic bath (KISS K6 from Huber, dimensions $210 \times 400 \times 546 \mathrm{~mm}$, heating capacity $2 \mathrm{~kW}$, bath volume $4.5 \mathrm{~L}$ ). The temperature of this bath is controlled with a precision of $0.1^{\circ} \mathrm{C}$ and is using glycol as the heat-carrying fluid. At each temperature, we let enough time pass ( $>30$ minutes) for the system to stabilize. A check of the temperature stabilization is done through repeated in-phase conductivity measurements. The sample a)
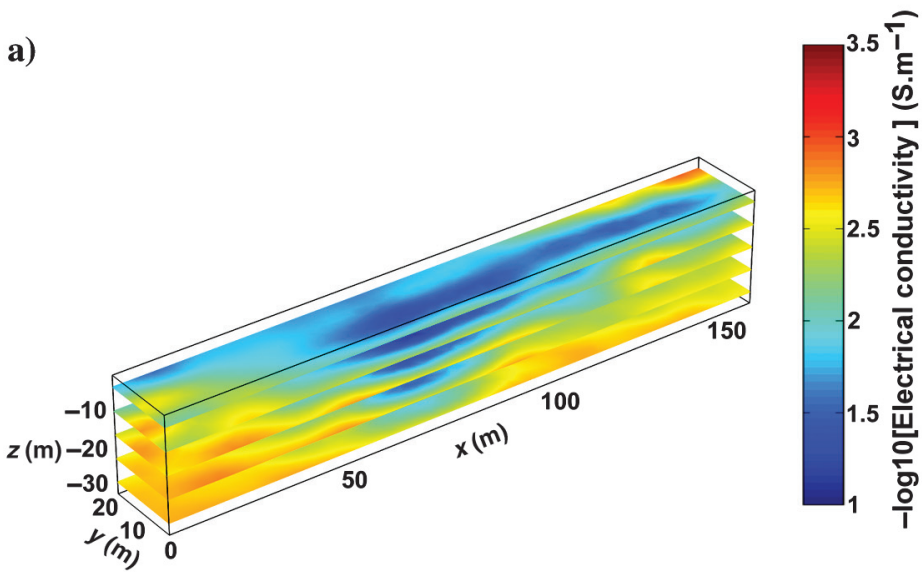

c)

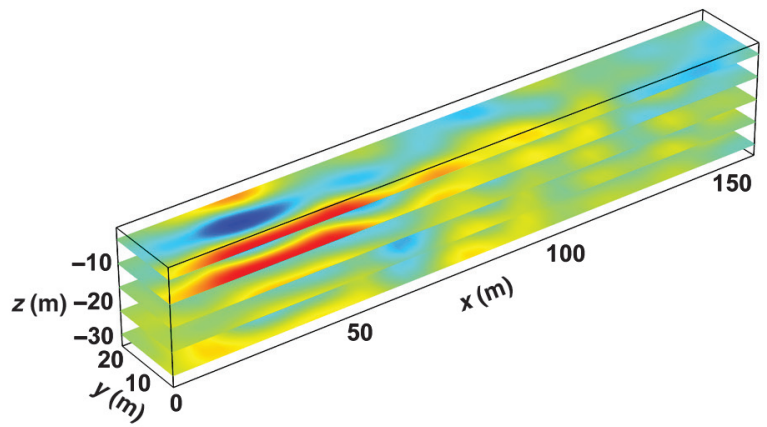

\section{b)}

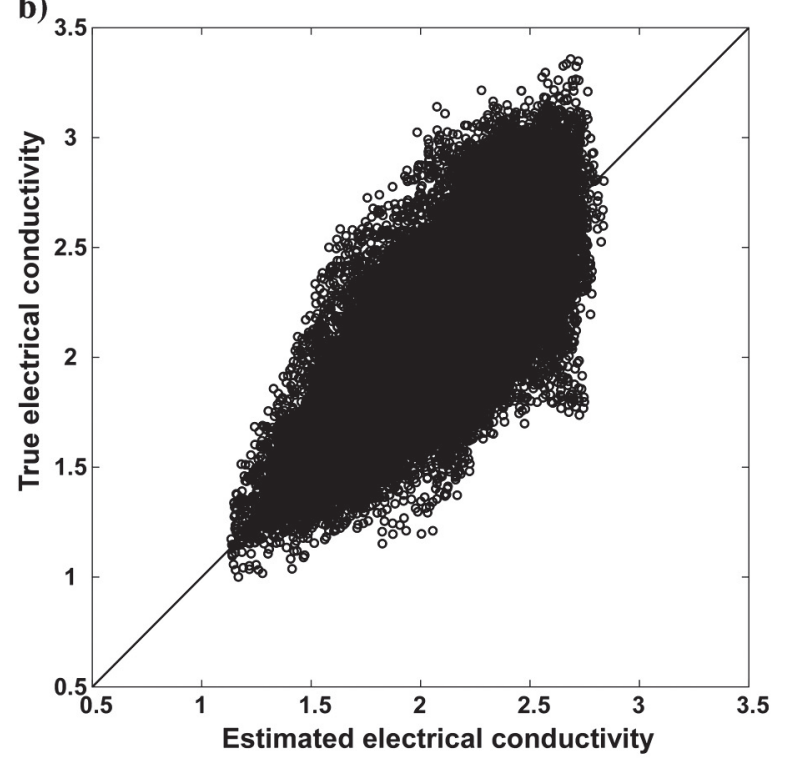

d) 1

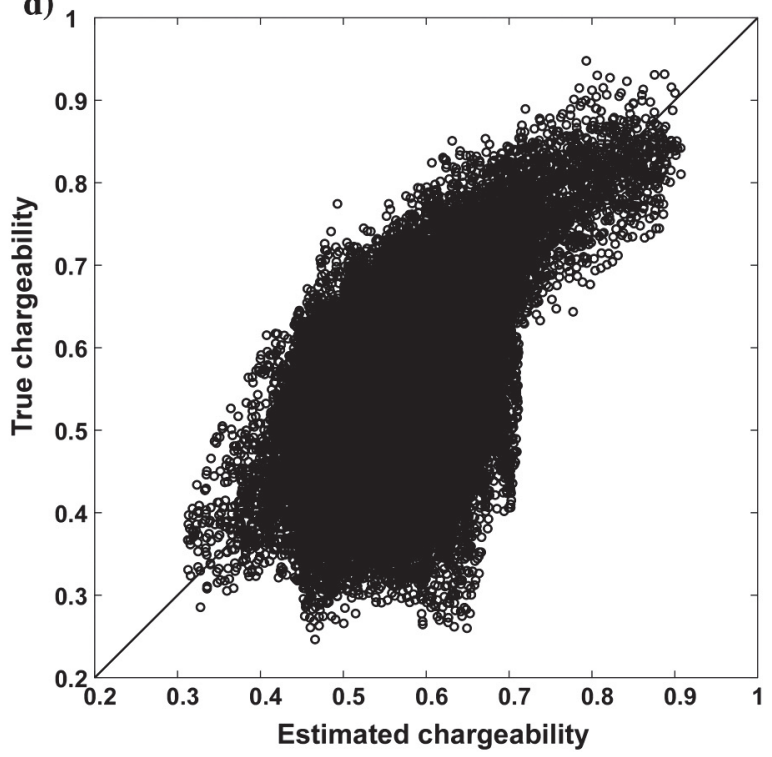

Figure 6. Tomography results of test 2 using the PCGA. (a) Estimated electrical conductivity field tomogram. (b) Scatterplot of the true electrical conductivity field against the estimated one. (c) Estimated chargeability field tomogram. (d) Scatterplot of the true chargeability field against the estimated one. 
is first saturated under vacuum with tap water with conductivity of $0.0715 \mathrm{~S} / \mathrm{m}$ at $25^{\circ} \mathrm{C}$. The voltage used in the experiment is $1 \mathrm{~V}$. The complex conductivity spectra are shown in Figure 8. The in-phase and quadrature conductivities (at $10 \mathrm{~Hz}$ ), the normalized chargeability, (computed as the difference of the in-phase conductivity between $0.1 \mathrm{~Hz}$ and $10 \mathrm{kHz}$ ), and the phase are shown as a function of the temperature in Figures 9 and 10.

To fit the data, we use an Arrhenius equation:

$$
\begin{aligned}
& \sigma^{\prime}(T)=\sigma_{\mathrm{o}}^{\prime} \exp \left(-\frac{E_{a}^{R}}{R T}\right), \\
& \sigma^{\prime \prime}(T)=\sigma_{\mathrm{o}}^{\prime \prime} \exp \left(-\frac{E_{a}^{I}}{R T}\right),
\end{aligned}
$$
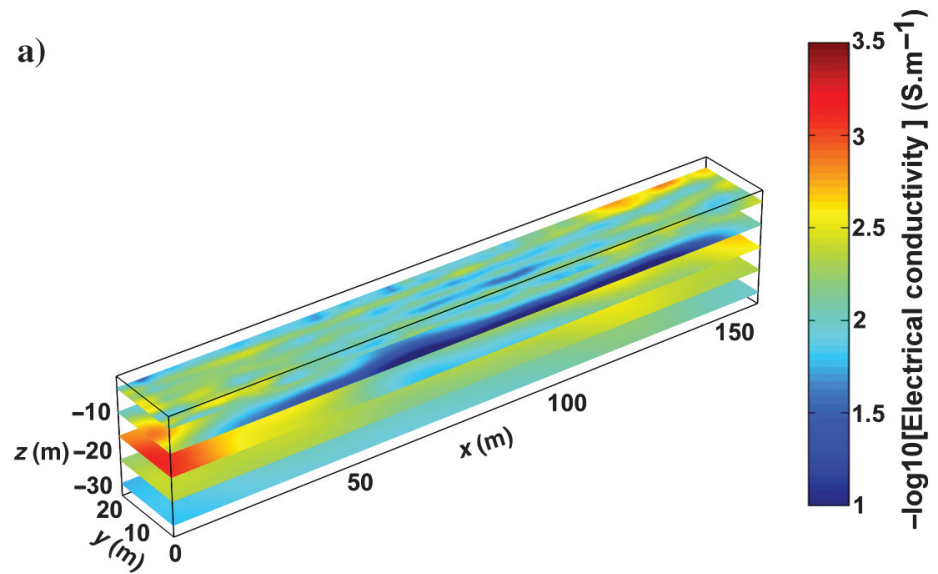

where $T$ and $T_{0}$ are expressed in degrees Kelvin $(\mathrm{K})\left(T_{0}=298 \mathrm{~K}\right)$, $\sigma_{\mathrm{o}}^{\prime}$ and $\sigma_{\mathrm{o}}^{\prime \prime}$ are two constants having the dimension of a conductivity, and $E_{a}^{R}$ and $E_{a}^{I}$ denote the activation energy associated with the real (in-phase) and imaginary components of the complex conductivity of the coal. The fit is shown in Figures 8 and 9 for the in-phase conductivity, the quadrature conductivity, and the normalized chargeability. The in-phase and quadrature conductivities have roughly the same activation energy (Figure 8). This value (approximately $15 \mathrm{~kJ} / \mathrm{Mol}$ ) is similar to the activation energy of the viscosity of the bulk pore water indicating that the conduction and polarization processes are related to the mobility of ionic charge carriers and their accumulation at the grain scale. Indeed, the viscosity and ionic mobility (alternatively the diffusion coefficients) are related to each other by the Einstein-Smoluchowski relationship.

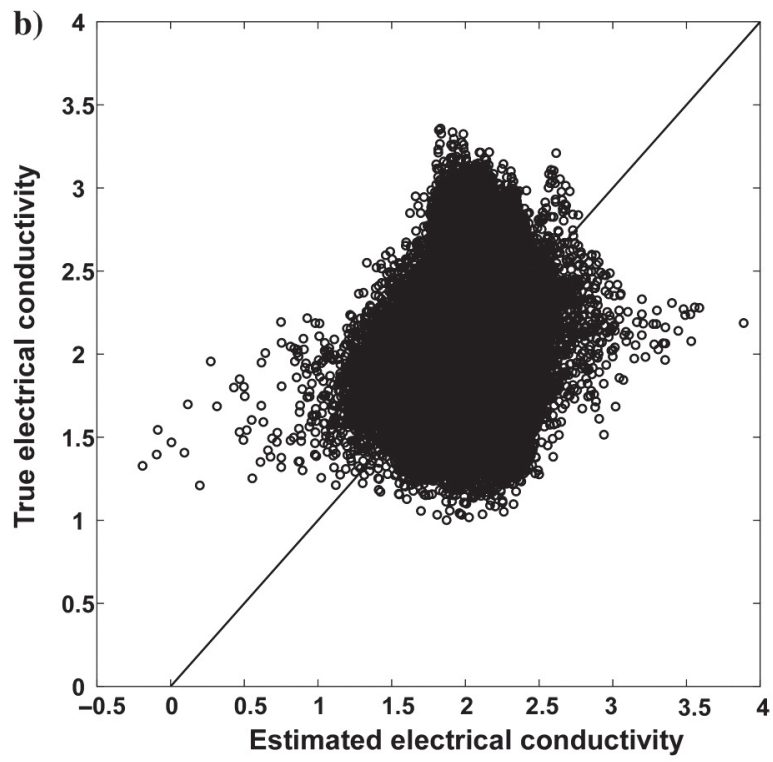

c)

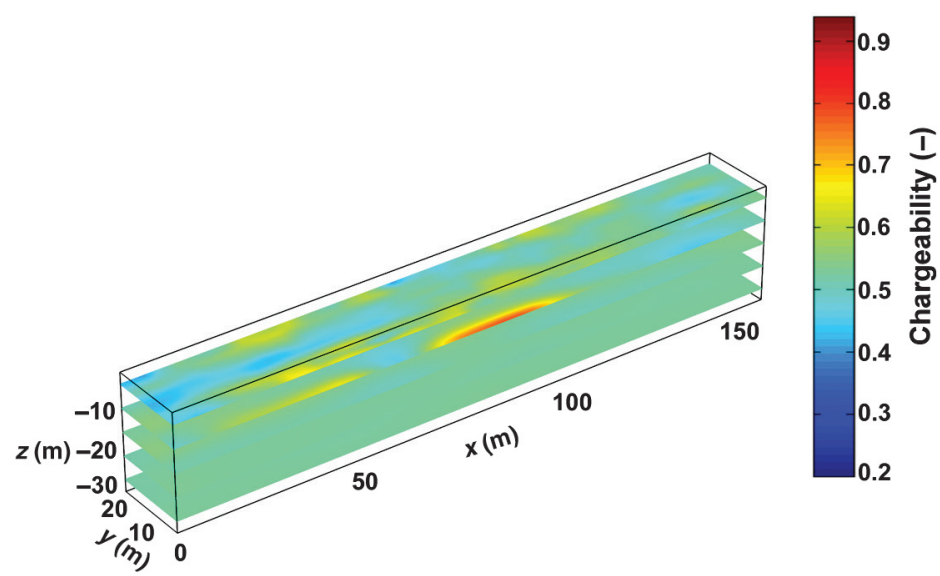

d)

Figure 7. Tomography results of test 2 using the GN method. (a) Estimated electrical conductivity field tomogram. (b) Scatterplot of the true electrical conductivity field against the estimated one. (c) Estimated chargeability field tomogram. (d) Scatterplot of the true chargeability field against the estimated one. 
It should be remembered that coal is characterized by a high cation exchange capacity (Wong et al., 1996). The results indicate that the normalized chargeability increases substantially with temperature. Hydrated coal in the vicinity of the burning front of the coal seam is therefore expected to be characterized by high values of the normalized chargeability. This implies that induced polarization is expected to be a pertinent method to localize the burning front of coal seams. This will be shown in the next section.

\section{FIELD APPLICATION}

\section{Description of the case study}

We now apply the PCGA algorithm to a case study of a coal seam fire. Our test site corresponds to the Lewis coal seam fire located northeast of the intersection of Highway 170 (Marshall Road) and Cherryvale Road, south of Boulder, Colorado (USA). The Lewis

Table 3. Data and parameters coefficients of determination $\left(R^{2}\right)$ values of the synthetic and Lewis fire study cases.

\begin{tabular}{|c|c|c|c|c|c|c|c|c|c|}
\hline & \multicolumn{6}{|c|}{ Data } & \multicolumn{3}{|c|}{ Parameters } \\
\hline & \multicolumn{2}{|c|}{ Test 1} & \multicolumn{2}{|c|}{ Test 2} & \multicolumn{2}{|c|}{ Lewis } & \multicolumn{2}{|c|}{ Test 1} & Test 2 \\
\hline & $\overline{\mathrm{GA}}$ & GN & GA & GN & GA & GN & GA & GN & GA GN \\
\hline$\sigma$ or $\sigma_{a}$ & 0.99 & 0.99 & 0.98 & 0.85 & 0.7 & 0.8 & 0.80 & 0.79 & $0.62 \quad 0.12$ \\
\hline$\eta$ or $\eta_{a}$ & 0.93 & 0.93 & 0.98 & 0.95 & 0.2 & 0.0193 & 0.52 & 0.39 & $\begin{array}{lll}0.30 & 0.07\end{array}$ \\
\hline
\end{tabular}

The quantity $\eta_{a}$ denotes the apparent chargeability, $\sigma_{a}$ denotes the apparent electrical conductivity, $\sigma$ denotes the electrical resistivity (in ohm-m), and $\eta$ denotes the chargeability (composed between 0 and 1 , dimensionless).In case of data, $R^{2}$ is computed for $\sigma_{a}$ and $\eta_{a}$. When considering the parameters, $R^{2}$ is computed from the estimated and true fields.

Table 4. Time domain induced polarization setup.

Amplitude of injected current

Injection duration

$100 \mathrm{~mA}$

Number of windows

Window duration

$0.1 \mathrm{~s}$

Table 5. Computational times (in h) for the synthetic case studies. Each duration represents the total computational time required for performing the corresponding tomography. All simulations were performed on a small workstation equipped with 32-core 2.1-3 GHz processors, 128 GB RAM and with no parallelization for the inverse problem.

Test 1 Test 2 Lewis fire

\begin{tabular}{lcccccc}
\cline { 2 - 6 } & GA & GN & GA & GN & GA & GN \\
\hline$\sigma$ - Tomography & 2.9 & 42.4 & 7.4 & 42.4 & 3.4 & 22.9 \\
$\eta-$ Tomography & 2.7 & 41.3 & 7.3 & 41.1 & 2.7 & 11.5 \\
\hline
\end{tabular}

mine was operated by the Peerless Coal Company from 1914 to 1942 (Rodriguez, 1983). The coal seam fire has been active for more than 50 years and is still ongoing. The coal seam fire is located in the Gorham subbituminous coal formation (2-4 m thick, Figure 11a),
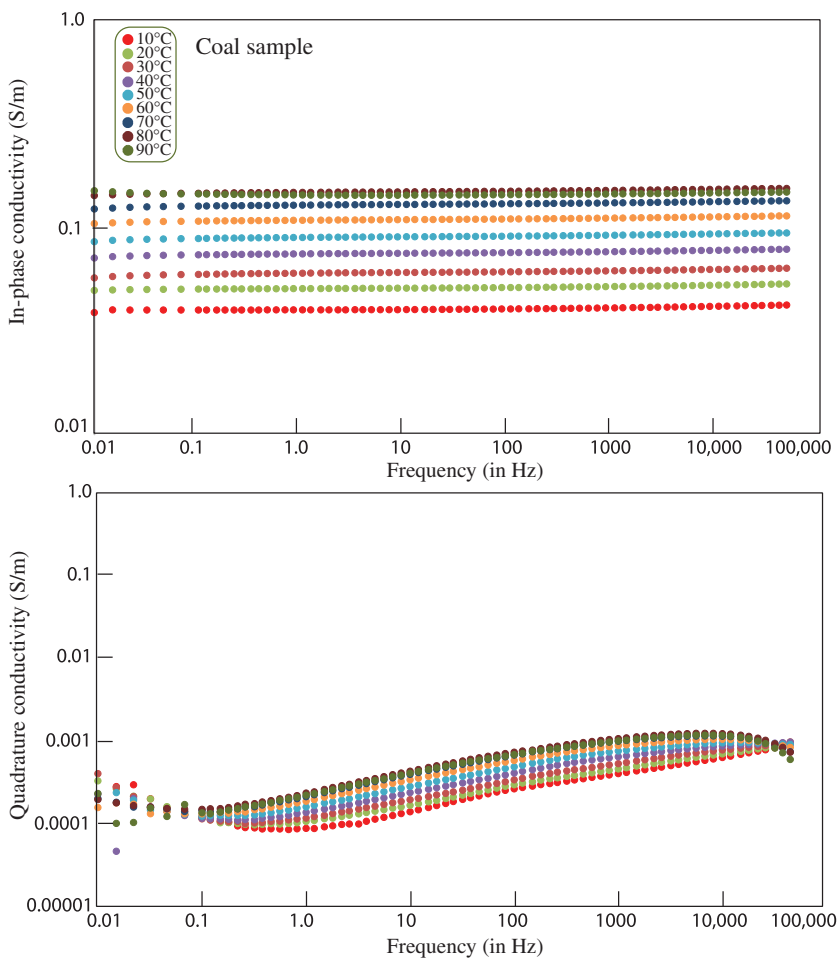

Figure 8. Complex conductivity spectra of a granular coal in tap water for the temperature range $10^{\circ} \mathrm{C}-90^{\circ} \mathrm{C}$. Note the increase of the in-phase and quadrature conductivities with the temperature.

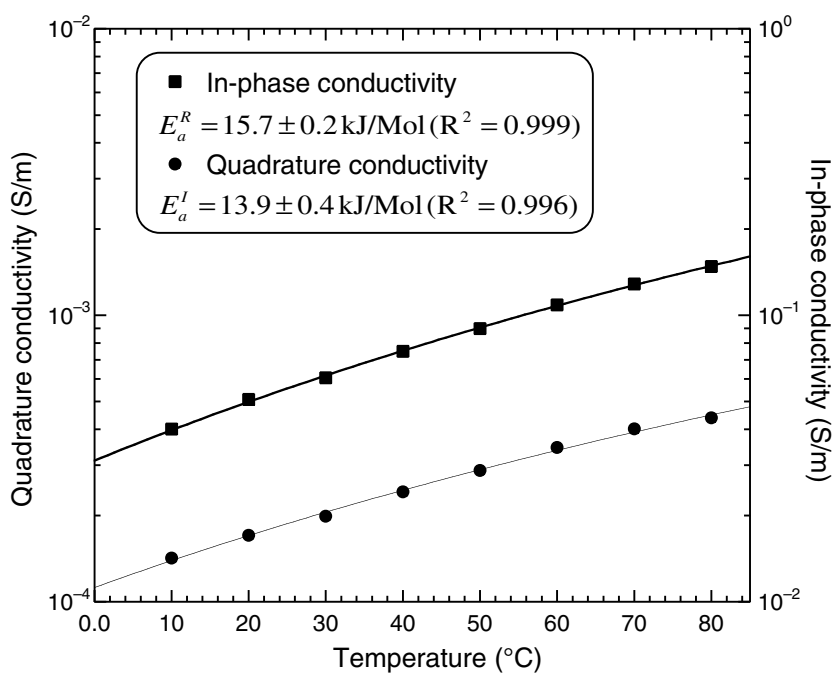

Figure 9. In-phase and quadrature conductivity at $10 \mathrm{~Hz}$ as a function of the temperature $\left(\right.$ in $^{\circ} \mathrm{C}$ ). The plain lines correspond to a fit of the experimental data with Arrhenius's law. The activation energies for the in-phase and quadrature conductivities are similar to the activation energy of the viscosity of water. This is expected because the mobility of the ions and counterions is related to the viscosity of water. 
lying roughly at a depth of $10-15 \mathrm{~m}$. The formation dips $4^{\circ}$ and is located above the Laramie Formation (Rushworth et al., 1989).

To better localize the coal seam fire, we performed a series of time-domain induced-polarization measurements. The inducedpolarization measurements were performed on five parallel profiles (see Figures $11 \mathrm{~b}$ and 12a) with spacing of $5 \mathrm{~m}$. A total of 32 stainless steel electrodes ( $5 \mathrm{~m}$ spacing between the electrodes along the profile) connected to an ABEM SAS4000 resistivity meter were used for each profile. A Wenner- $\alpha$ configuration was used to collect 629 apparent resistivities, for which a current of $100 \mathrm{~mA}$ is injected between the current electrodes $\mathrm{A}$ and $\mathrm{B}$ and the voltage is measured between the $\mathrm{M}$ and $\mathrm{N}$ potential electrodes, which are located between the $[\mathrm{A}, \mathrm{B}]$ current electrodes (Wenner array).

For the chargeabilities, the primary current was injected for $1.6 \mathrm{~s}$. The decay of the secondary voltage over time is measured over 10 windows of $0.1 \mathrm{~s}$ each (10 partial chargeabilities are measured; see Figure 12 and Table 4). We use a dead time of $400 \mathrm{~ms}$ after the shutdown of the primary current. In the laboratory with small cables, we observed electromagnetic inductive and capacitive coupling effects for a dead time of $35 \mathrm{~ms}$ (Mao and Revil, 2016, their Figure 15). For field experiments, depending on the length of the cables, Kemna et al. (2012) recommend a dead time of 200-400 ms if the same cables are used for the current injection and voltage measurements to avoid capacitive and inductive effects. To give an idea about the chargeability data, we plot in Figure 12b the evolution of the partial chargeabilities over time for two quadrupoles $[\mathrm{A}, \mathrm{B}, \mathrm{M}, \mathrm{N}]$ on profile 1 .

The data standard deviations are used to build the data covariance matrix (diagonal matrix with the inverse of the standard deviations in its diagonal). The apparent resistivities range from 8.9 to $1267 \mathrm{ohm}-\mathrm{m}$ and have a mean value of $148 \mathrm{ohm}-\mathrm{m}$. The apparent chargeabilities range from 0.19 to $237.94 \mathrm{mV} \mathrm{V}^{-1}$ and have a mean of $51.9 \mathrm{mV} \mathrm{V}^{-1}$.

\section{Determination of the semivariogram}

We need to deal with the issue of characterizing a variogram that will geostatistically guide the inversion by providing physically

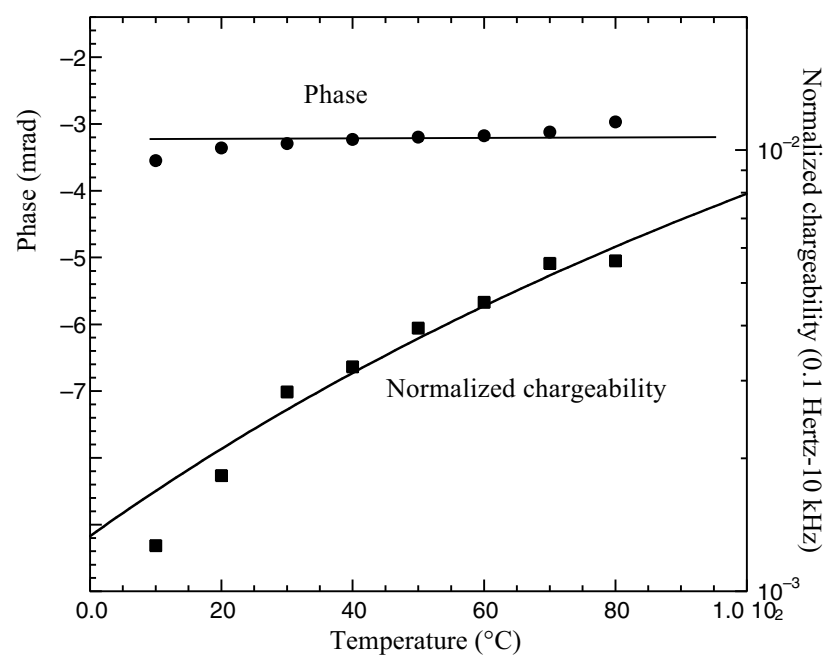

Figure 10. Phase and normalized chargeability (between 0.1 Hertz and $10 \mathrm{kHertz}$ ) as a function of the temperature (in ${ }^{\circ} \mathrm{C}$ ). The phase is roughly independent of the temperature, whereas the noralized chargeability data can be fitted by Arrhenius's law (plain line) with an activation energy of $15.1 \pm 1.4 \mathrm{~kJ} / \mathrm{Mol}\left(R^{2}=0.96\right)$. meaningful constraints. In practice, when we do not have any prior information about the field, we can consider that the parameters are known at some few discrete spatial locations (e.g., through borehole data). Then a weighted least-squares minimization is used to estimate the parameters of an experimental variogram that best fits these data. Nevertheless, it may be sometimes quite challenging to collect samples at different depths. In this case, it is judicious to derive the experimental variogram from a parameter that gives direct information on the parameter that we want to estimate. For instance, in our case, we used the self-potential (SP) data to estimate an experimental variogram for the conductivity and chargeability fields. Because the SP field is related to the presence of the burning front (through a thermoelectric effect), it can be used to infer statistical information on its spatial structures at least in the horizontal plane. The idea of using theoretical variograms to fit experimental ones is not new in geosciences (e.g., Deutsch and Journel, 1992; Goovaerts, 1997; Kitanidis, 1997; Webster and Oliver, 2001; Minsasny and McBratney, 2005). Several types of variograms can be used to fit the data (e.g., linear, exponential, Gaussian spherical). We found that an exponential variogram best described the behavior of our SP data. The variogram that we used is defined as follows:
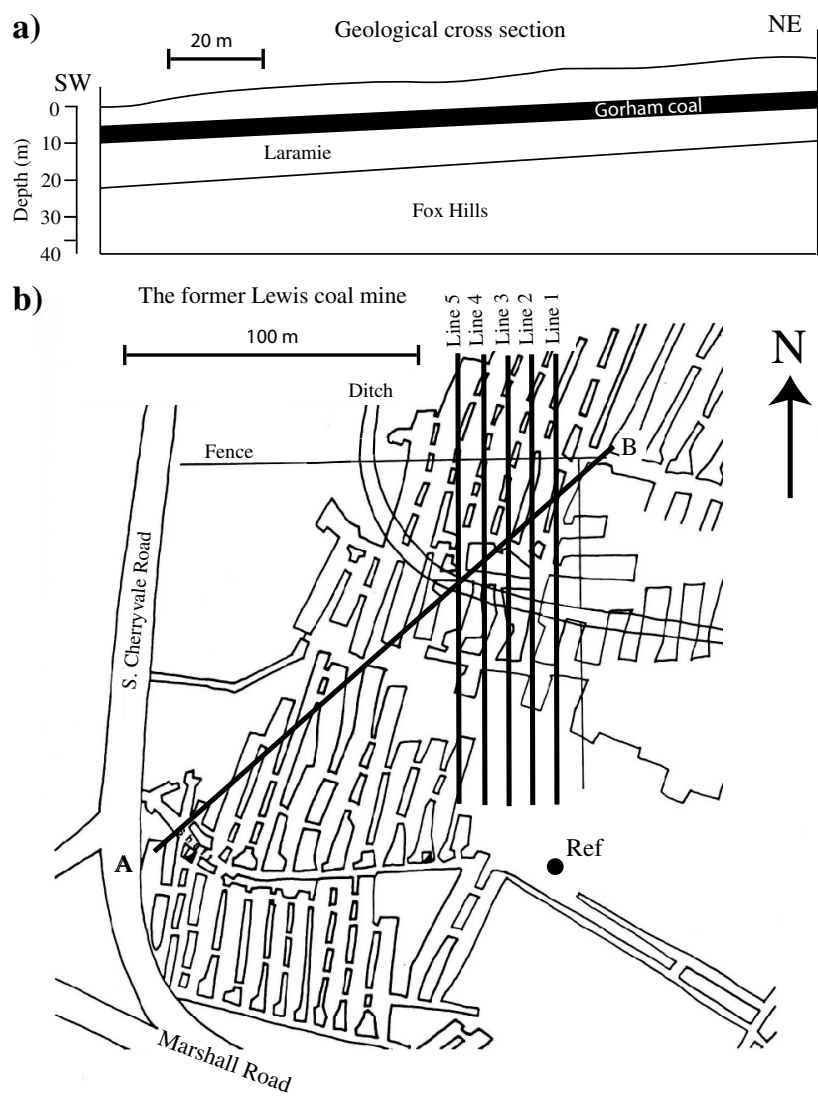

Figure 11. Geologic cross section and map of the Lewis coal mine (from Revil et al., 2013). (a) Position of the Gorham coal seam. (b) Map of the former Lewis coal mine. The five lines (P1-P5) represent the profiles used for acquiring resistivity and time-domain induced-polarization measurements in order image the electrical resistivity and chargeability fields. Ref represents the reference for the self-potential survey discussed later in the paper (see Figure 14). 


$$
\gamma(h)=\sigma^{2}\left(1-\exp \left(-\sqrt{\left(\frac{h_{x}}{l_{x}}\right)^{2}+\left(\frac{h_{y}}{l_{y}}\right)^{2}+\left(\frac{h_{z}}{l_{z}}\right)^{2}}\right)\right)
$$

where $h=\left(h_{x}, h_{y}, h_{z}\right)$ is the $3 \mathrm{D}$ direction vector and $l_{x}, l_{y}$, and $l_{z}$ are the correlations lengths in the $x$-, $y$-, and $z$-directions. The estimated values of $\sigma^{2}, l_{x}, l_{y}$, and $l_{z}$ are reported in Table 1 .

\section{Result of the inversion and interpretation}

We seek to invert the field data using the PCGA approach. Our field is discretized into 48,000 unknown cells, the truncation order $K=30$, and the theoretical variograms used for the electrical resistivity and the chargeability models are exponential. A complete description of the inversion parameters is reported in Table 1. We use the geometric mean of the apparent electrical resistivities as the starting initial resistivity model.

In this case, we used $K=30$. The relative errors $\lambda_{K+1} / \lambda_{1}$ associated to the electrical resistivity and chargeability covariance matrices are 0.0033 and 0.02 . The inverted electrical resistivity tomogram is shown in Figure 13. It shows the presence of a very low resistivity anomaly $\approx 1.6 \mathrm{ohm}-\mathrm{m}$, which has a depth starting from approximately $13 \mathrm{~m}$ above the ground surface and extends from $x=50 \mathrm{~m}$ to $x=70 \mathrm{~m}$. Duba (1977) states that coked coal can have a very low
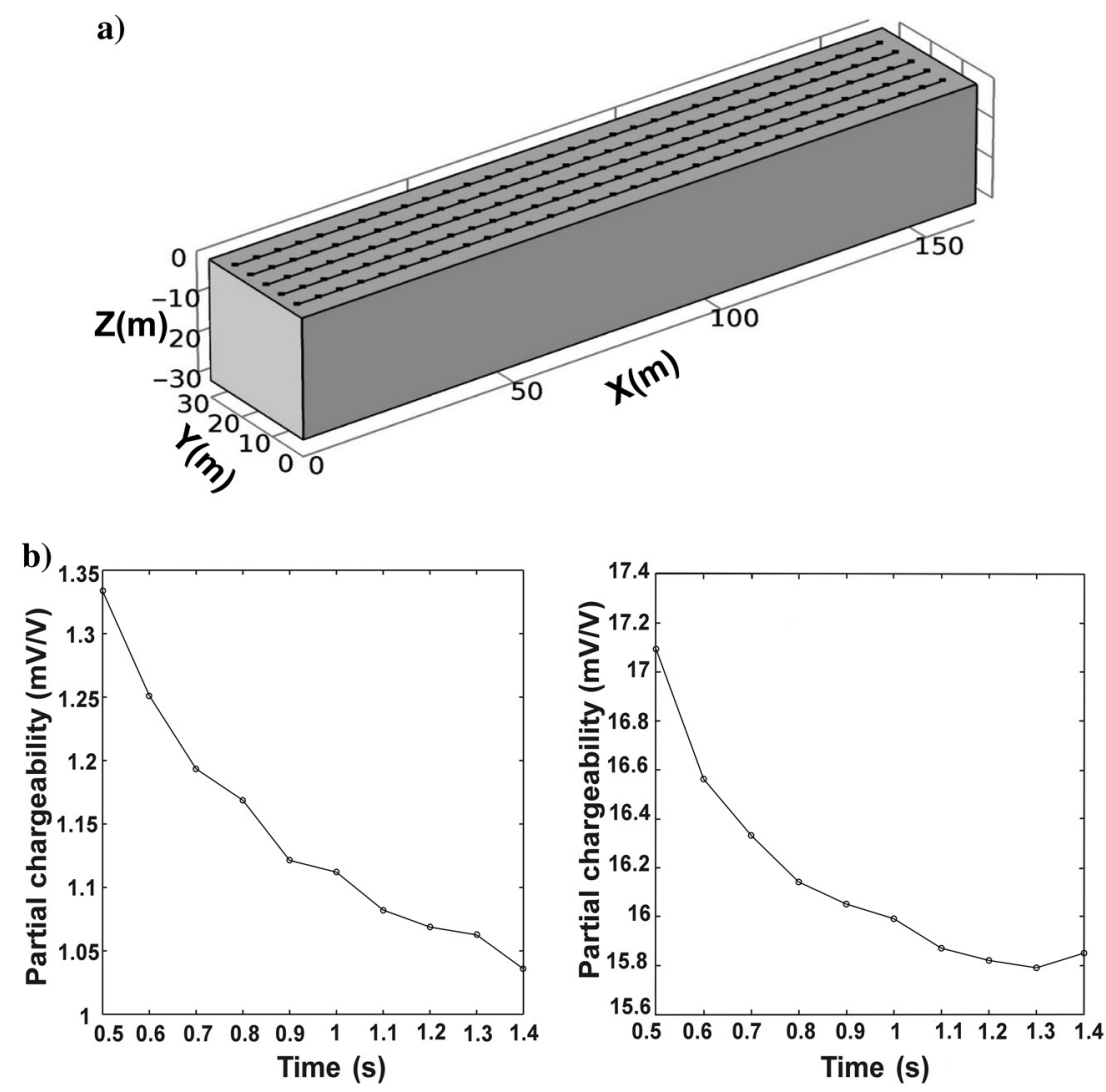

Figure 12. Simulation domain and data. (a) 3D representation of the simulation domain. (b) Example of chargeability decays on two quadrupoles. The region of interest represents a 3D volume of $162 \times 20 \times 30 \mathrm{~m}$, the five profiles used for collecting induced polarization measurements are, respectively, located at $y=0,5,10,15$, and $20 \mathrm{~m}$ and are composed of 32 electrodes each. resistivity $(<1 \mathrm{ohm}-\mathrm{m})$, which can suggest that the conductive body that we are seeing in our 3D resistivity tomography potentially corresponds to the burning region. In addition, the reports of the inspections made by the Colorado Geological Survey (CGS) and the Colorado Inactive Mine Reclamation Program (CIMRP) in 1984 and 1988 are in accordance with the location of the imaged anomaly.

We now use the electrical resistivity field illustrated in Figure 13 to compute the apparent chargeabilities using equation 7 . The initial chargeability model that we used for running the inversion is given by

$$
\eta_{0}=\frac{\eta_{n}}{\sigma}
$$

where $\sigma$ is the electrical conductivity and $\eta_{n}$ is the normalized chargeability. The inversion process remains unchanged, and the best intrinsic chargeability model obtained after five iterations of the inverse algorithm is represented in Figure 13c. It shows a region with a chargeability of approximately 0.15 coinciding with the area where we observed the low resistivity body and that is plausibly the burning region.

We combine the resistivity and chargeability data through a normalized burning front index (BFI) (dimensionless), which is nothing else that the normalized chargeability is itself normalized to its highest value:

$$
\mathrm{BFI}=\left(\frac{\eta}{\eta_{\max }}\right)\left(\frac{\sigma}{\sigma_{\max }}\right)
$$

where $\sigma$ denotes the electrical conductivity, $\sigma_{\max }$ is the highest value of the of the conductivity field, $\eta$ is the intrinsic chargeability, and $\eta_{\max }$ is the highest value of the chargeability field. The normalized burning front index is between 0 and 1 and is presented in Figure 13e. It clearly exhibits an area of high values corresponding to the position of the target, which is the burning region in our case. It shows that the anomaly starts at a depth of approximately $13 \mathrm{~m}$ (located in the coal seam formation); the position of this anomaly is consistent with the self-potential anomaly of thermoelectric origin recorded at the ground surface (see Figure 14). Therefore, the BFI combined the information from electrical resistivity with the one obtained from the chargeability to better diagnose, identify, and delineate the location of the coal seam fire.

For comparison purposes, we also performed the inversion of the field data using the GN method. The results of this inversion are illustrated in Figure 13. As one can see, the GN method fairly reproduces the main heterogeneities of the resistivity field (Figure 13b). That said, the anomaly associated to the burning coal stretches down and is not constrained to the coal layer as obtained with the PCGA. The chargeability field is globally similar to the one obtained with the PCGA, but it seems to have slightly lower magnitude values (Figure 13d). Although the BFI clearly reveals the presence of an anomaly related 
to burning coal (Figure 13f), the delineation and the magnitudes of the BFI are questionable. In fact, the GN tends to oversmooth the anomaly and to make it extend outside the coal formation. These results would be probably much better if more measurements were available to better constrain the inverse problem. The normalized chargeability defined by equation 30 is also normalized with respect to its maximum value to get an index between 0 and 1 . For porous rocks without metallic particles, the normalized chargeability is con- trolled by the cation exchange capacity of the material (the charge of the surface per unit mass of grains) and a mobility of the charge carriers (for soils, see Revil et al., 2017a). Coal is characterized by a high cation exchange capacity, as mentioned previously. Therefore, coal is expected to be associated with a high normalized chargeability. Because the normalized chargeability increases linearly with temperature as discussed above (see also Revil et al., 2017a), we expect that burning coal is associated with a high normalized chargeability. a)

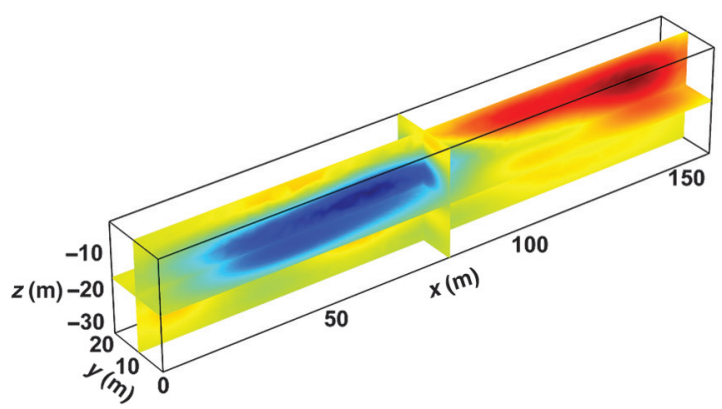

c)

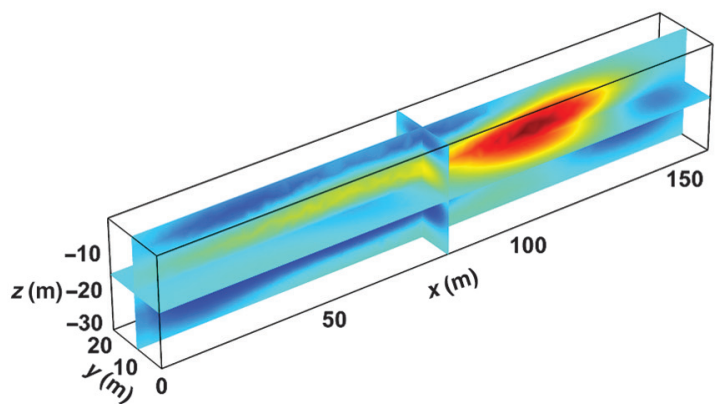

e)

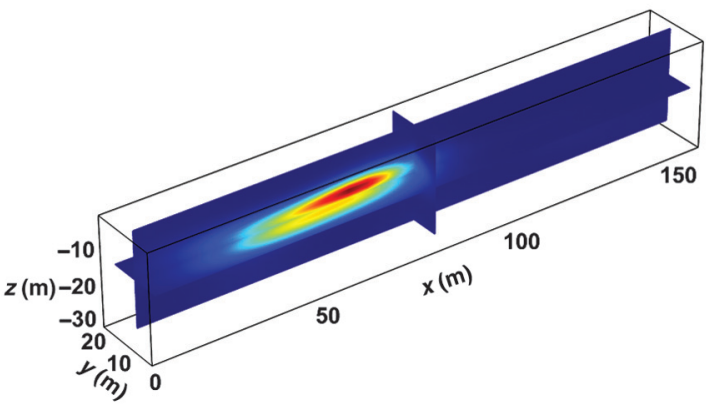

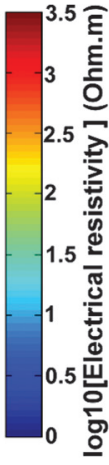

b)

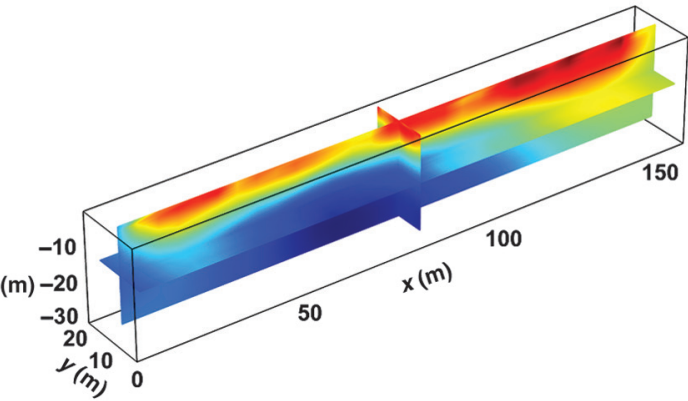

d)
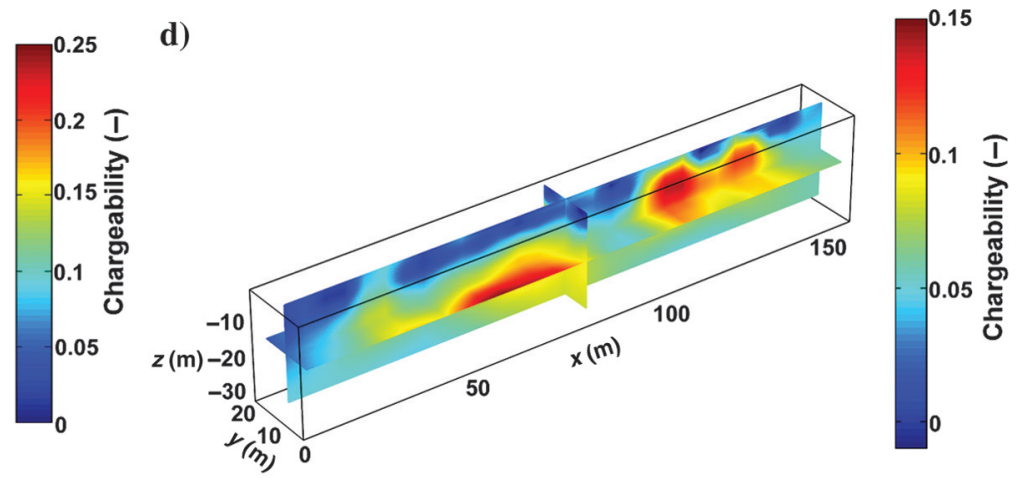

f)

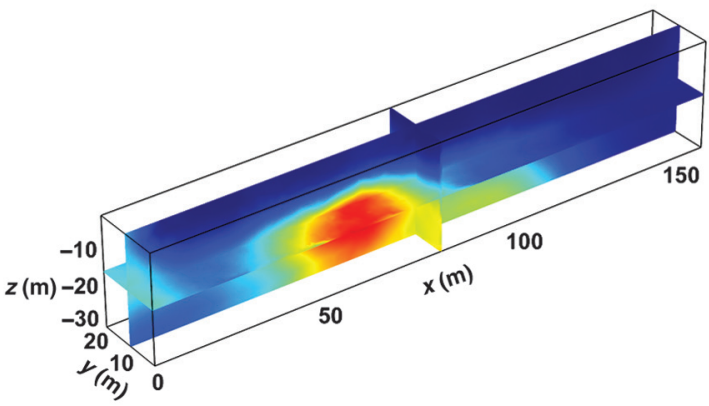

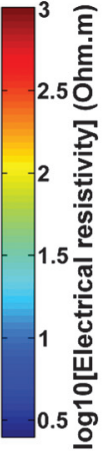

톨
告

Figure 13. Field data results. (a) Inverted 3D electrical conductivity spatial distribution obtained with PCGA. (b) Inverted 3D electrical conductivity spatial distribution obtained with GN. (c) Inverted 3D chargeability field obtained with PCGA. (d) Inverted 3D chargeability field obtained with GN. (e) PCGA BFI field. (f) GN BFI field. For the PCGA and GN tomograms, the electrical conductivity tomogram shows a region of very low resistivity, which plausibly coincides with the localization of the burning front. That said, PCGA better delineates this anomaly because it vertically restricts it to the coal layer, whereas GN tends to oversmooth this anomaly. The chargeability is high for the coal seam and the burning front of the coal seam. This is why the anomalous chargeability distribution is broader than for the resistivity. The 3D BFI determination shows the area of high probability of the presence of the coal fire. This area seemingly starts at a depth of approximately $13 \mathrm{~m}$ and extends although the simulation domain from $x=50 \mathrm{~m}$ to $x=70 \mathrm{~m}$. This result is consistent with the self-potential map shown in Figure 14. 


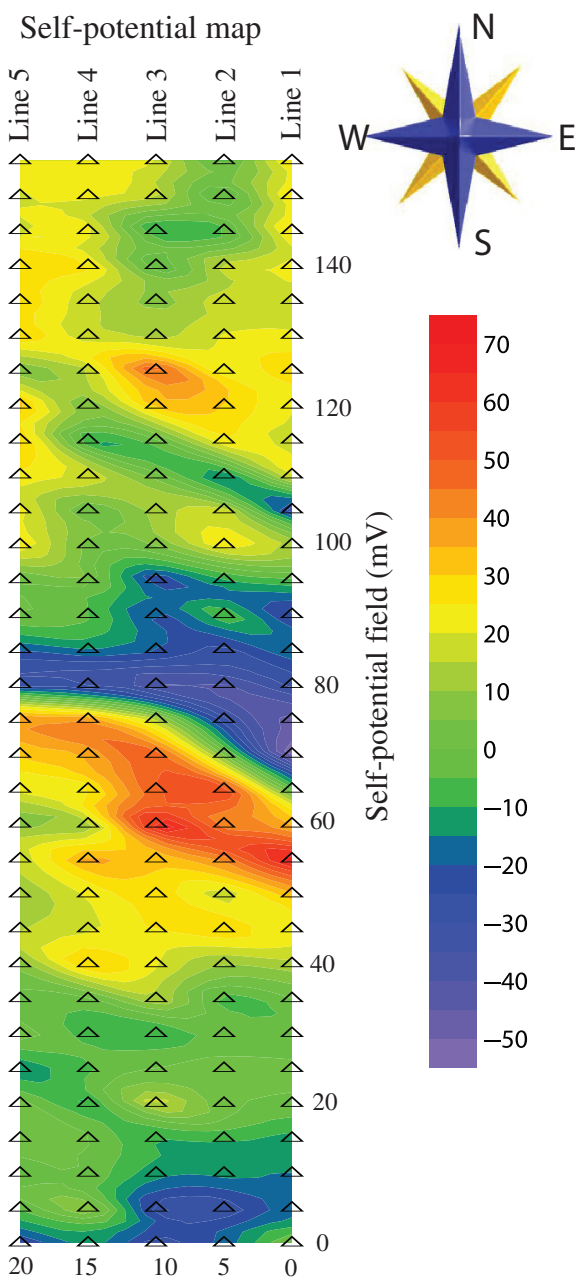

Figure 14. Self-potential map. The position of the reference is shown in Figure 5. The five profiles used for the induced polarization survey are denoted P1, P2, P3, P4, and P5, whereas the triangles denote the locations of the 160 electrodes. Each profile is $5 \mathrm{~m}$ apart from the other, and the electrodes interspace is $5 \mathrm{~m}$. We observe in the middle of the domain, a negative self-potential anomaly (approximately $-50 \mathrm{mV}$ ) of which position is consistent with the high BFI values retrieved from our 3D tomograms (see Figure 13). This negative anomaly is likely associated with a thermoelectric effect (see Revil et al., 2016).

\section{CONCLUSION}

We used a geostatistical inversion approach called the principal component geostatistical approach (PCGA) to invert time-domain induced-polarization data. The major advantages of this technique include (1) the reduction of the dimensionality of the inverse problem by factorizing the prior covariance matrix as a product of lowrank matrices and (2) avoiding the direct assembling of the Jacobian matrix. These two points make the PCGA an appealing technique from a computational point of view. Three case studies have been investigated. In the first two cases, we successfully recovered the spatial heterogeneities of the electrical conductivity and intrinsic chargeability fields of a 3D synthetic model. We compared our inverse methodology to the conventional Gauss-Newton (GN) method, which uses the first-order derivatives as smoothing constraints, and we showed that using the geostatistical constraints can improve reconstruction accuracy. In third case, we invert the electrical resistivity and chargeability for a field survey performed over the Lewis coal seam in Colorado. A burning front index combining the chargeability and electrical resistivity tomograms derived from our algorithm provides a 3D image of the burning front. Laboratory experiments performed at lower temperatures (up to $90^{\circ} \mathrm{C}$ ) support the results of such tomography. This algorithm and methodology could be applied to other problems such as $\mathrm{CO}_{2}$ sequestration to image the evolution of the $\mathrm{CO}_{2}$ plume. We expect also to apply this methodology to the full-waveform inversion of time-domain induced-polarization data.

\section{ACKNOWLEDGEMENTS}

We thank the editor $\mathrm{C}$. Farquharson and the three referees for their very constructive comments of our manuscript. We also acknowledge the support of the University of Melbourne through a project funded by the Commonwealth of Australia (contract CR-2016-UNIV.MELBOURNE-147672-UMR5275).

\section{REFERENCES}

Attwa, M., and T. Günther, 2013, Spectral induced polarization measurements for environmental purposes and predicting the hydraulic conductivity in sandy aquifers: Hydrology and Earth System Sciences (HESS), 10, 5315-5354

Bakhos, T., M. Cardiff, W. Barrash, and P. K. Kitanidis, 2014, Data processing for harmonic pumping tests: Journal of Hydrology, 511, 310-319, doi: 10.1016/j.jhydrol.2014.01.007.

Barker, R. D., 1990, Investigation of groundwater salinity by geophysical methods, in S. H. Ward, ed., Geotechnical and environmental geophysics, Vol. II: SEG Investigations in Geophysics 5, 201-211.

Binley, A., and A. Kemna, 2005, DC resistivity and induced polarization methods, in Y. Rubin and S. S. Hubbard, eds., Hydrogeophysics: Water science and technology library: Springer.

Cardiff, M., W. Barrash, and P. K. Kitanidis, 2012, A field proof-of-concept of aquifer imaging using 3-D transient hydraulic tomography with modular, temporarily-emplaced equipment: Water Resources Research, 48, W05531, doi: 10.1029/2011WR011704

Cardiff, M., W. Barrash, and P. K. Kitanidis, 2013, Hydraulic conductivity imaging from 3-D transient hydraulic tomography at several pumping/ observation densities: Water Resources Research, 49, 7311-7326, doi: 10.1002/wrcr.20519.

Carlson, N., and K. L. Zonge, 1996, Induced polarization effects associated with hydrocarbon accumulations: minimization and evaluation of cultural influences, in D. Schumacher and M. A. Abrams, eds., Hydrocarbon migration and its near-surface expression: AAPG Memoir 66, 127-137.

Cirpka, O. A., and P. K. Kitanidis, 2000, Sensitivity of temporal moments calculated by the adjoint-state method and joint inversing of head and tracer data: Advances in Water Resources, 24, 89-103, doi: 10.1016/ S0309-1708(00)00007-5.

Cosenza, P., S. Hedan, and V. Valle, 2016, Caractérisation non destructive de la zone endommagée des galeries souterraines excavées dans les roches argileuses: Revue Fraçaise de Géotechnique, 148, 22.

Dahlin, T., and V. Leroux, 2012, Improvement in time-domain induced polarization data quality with multi-electrode systems by separating current and potential cables: Near Surface Geophysics, 10, 545-565, doi: 10 3997/1873-0604.2012028.

Deutsch, C., and A. Journel, 1992, GSLIB: Geostatistical software library and user's guide: Oxford University Press.

Duba, A. F., 1977, Electrical conductivity of coal and coal char: Fuel, 56, 441-443, doi: 10.1016/0016-2361(77)90074-6.

Florsch, N., M. Llubes, F. Téreygeol, A. Ghorbani, and P. Roblet, 2011, Quantification of slag heap volumes and masses through the use of induced polarization: Application to the Castel-Minier site: Journal of Archaeological Science, 38, 438-451, doi: 10.1016/j.jas.2010.09.027.

Gazoty, A., G. Fiandaca, J. Pedersen, E. Auken, A. V. Christiansen, and J. K. Pedersen, 2012, Application of time domain induced polarization to the mapping of lithotypes in a landfill site: Hydrology and Earth System Sciences, 16, 1793-1804, doi: 10.5194/hess-16-1793-2012.

Goovaerts, P., 1997, Geostatistics for natural resources evaluation: Oxford University Press. 
Gurin, G. A. Tarasov, Y. Ilyin, and K. Titov, 2013, Time domain spectral induced polarization of disseminated electronic conductors: Laboratory data analysis through the Debye decomposition approach: Journal of Applied Geophysics, 98, 44-53, doi: 10.1016/j.jappgeo.2013.07.008.

Johnson, T. C., R. J. Versteeg, M. Rockhold, L. D. Slater, D. Ntarlagiannis, W. J. Greenwood, and J. Zachara, 2012, Characterization of a contaminated wellfield using 3D electrical resistivity tomography implemented with geostatistical, discontinuous boundary, and known conductivity constraints: Geophysics, 77, no. 6, EN85-EN96, doi: 10.1190/ geo2012-0121.1.

Joseph, S., M. Ingham, and G. Gouws, 2016, Spectral induced polarization measurements on sieved sands and the relationship to permeability: Water Resources Research, 52, 4226-4246, doi: 10.1002/2015WR018087.

Karaoulis, M., A. Revil, D. D. Werkema, B. J. Minsley, W. F. Woodruff, and A. Kemna, 2011, Time-lapse three-dimensional inversion of complex conductivity data using an active time constrained (ATC) approach: Geophysical Journal International, 187, 237-251, doi: 10.1111/j.1365-246X .2011.05156.x

Kemna, A., A. Binley, G. Cassiani, E. Niederleithinger, A. Revil, L. Slater, K. H. Williams, A. Flores Orozco, F.-H. Haegel, A. Hördt, S. Kruschwitz, V. Leroux, K. Titov, and E. Zimmermann, 2012, An overview of the spectral induced polarization method for near-surface applications: Near Surface Geophysics, 10, 453-468, doi: 10.3997/1873-0604.2012027.

Kemna, A., A. Binley, and L. Slater, 2004, Crosshole IP imaging for engineering and environmental applications: Geophysics, 69, 97-107, doi: 10 $1190 / 1.1649379$.

Kingman, J. E. E., J. G. Donohue, and T. J. Ritchie, 2007, Distributed acquisition in electrical geophysical systems: in B. Milkereit, ed., Proceedings of Exploration 07: Fifth Decennial International Conference on Mineral Exploration, Decennial Mineral Exploration Conferences, 425-432.

Kitanidis, P. K., 1995, Quasi-linear geostatistical theory for inversing: Water Resources Research, 31, 2411-2419, doi: 10.1029/95WR01945.

Kitanidis, P. K., 1997, Introduction to geostatistics: Applications in hydrogeology: Cambridge University Press

Kitanidis, P. K., and J. Lee, 2014, Principal component geostatistical approach for large-dimensional inverse problems: Water Resources Research, 50, 5428-5443, doi: 10.1002/wrcr.v50.7.

Kitanidis, P. K., and J. H. Lee, 2015, Three-dimensional ERT imaging by the geostatistical approach: AGU Fall Meeting Abstracts.

LaBrecque, D. J., 1991, IP tomography: 61st Annual International Meeting, SEG, Expanded Abstracts, 413-416.

Lee, J., and P. K. Kitanidis, 2014. Large-scale hydraulic tomography and joint inversion of head and tracer data using the Principal Component Geostatistical Approach (PCGA): Water Resources Research, 50, 5410-5427, doi: 10.1002/wrcr.v50.7.

Leroux, V., T. Dahlin, and H. Rosqvist, 2010, Time-domain IP and resistivity sections measured at four landfills with different contents: 16th European Meeting of Environmental and Engineering Geophysics (Near Surface), Expanded abstracts, P09.

Li, Y., and D. W. Oldenburg, 2003, Fast inversion of large-scale magnetic data using wavelet transforms and a logarithmic barrier method: Geophysical Journal International, 152, 251-265, doi: 10.1046/j.1365246X.2003.01766.X

Linde, N., A. Binley, A. Tryggvason, L. B. Pedersen, and A. Revil, 2006, Improved hydrogeophysical characterization using joint inversion of crosshole electrical resistance and ground penetrating radar traveltime data: Water Resources Research, 42, W12404, doi: 10.029/ 2006WR005131.

Loke, M. H., and R. D. Barker, 1995, Least-squares deconvolution of apparent resistivity pseudosections: Geophysics, 60, 1682-1690, doi: 10.1190/1.1443900.

Loke, M. H., and R. D. Barker, 1996, Rapid least-squares inversion of apparent resistivity pseudosections by a quasi-Newton method: Geophysical Prospecting, 44, 131-152, doi: 10.1111/j.1365-2478.1996. tb00142.x.

Macnae, J., 2015, Quantifying airborne induced polarization effects in helicopter time domain electromagnetics: Journal of Applied Geophysics, 135, 495-502, doi: 10.1016/j.jappgeo.2015.10.016.

Macnae, J., 2016, Quantitative estimation of intrinsic induced polarization and superparamagnetic parameters from airborne electromagnetic data: Geophysics, 81, no. 6, E433-E446, doi: 10.1190/geo2016-0110.1.

Mao, D., and A. Revil, 2016, Induced polarization response of porous media with metallic particles - Part 3: A new approach to time-domain induced polarization tomography: Geophysics, 81, no. 4, D345-D357, doi: 10 $.1190 /$ geo2015-0283.1

Marchant, D., E. Haber, and D. W. Oldenburg, 2013, Inductive source induced polarization: Geophysical Journal International, 192, 602-612, doi: $10.1093 / \mathrm{gji} / \mathrm{ggs} 040$.

Marchant, D., E. Haber, and D. W. Oldenburg, 2014, Three-dimensional modeling of IP effects in time-domain electromagnetic data: Geophysics, 79, no. 6, E303-E314, doi: 10.1190/geo2014-0060.1.
Maurer, H., K. Holliger, and D. E. Boerner, 1998, Stochastic regularization Smoothness or similarity?: Geophysical Research Letters, 25, 2889-2892, doi: 10.1029/98GL02183.

Minsasny, B., and A. B. McBratney, 2005, The Matérn function as general model for soil variograms: Geoderma, 128, 192-207, doi: 10.1016/j. geoderma.2005.04.003.

Oldenburg, D. W., and Y. Li, 1994, Inversion of induced polarization data: Geophysics, 59, 1327-1341, doi: 10.1190/1.1443692.

Oldenburg, D. W., Y. Li, and R. G. Ellis, 1997, Inversion of geophysical data over a copper-gold porphyry deposit: A case history for Mt. Milligan: Geophysics, 62, 1419-1431, doi: 10.1190/1.1444246.

Olsson, P. I., G. Fiandaca, J. J. Larsen, T. Dahlin, and E. Auken, 2016, Doubling the spectrum of time-domain induced polarization by harmonic de-noising, drift correction, spike removal, tapered gating and data uncertainty estimation: Geophysical Journal of International, 207, 774-784, doi: $10.1093 / \mathrm{gji} / \mathrm{ggw} 260$.

Pelton, W. H., S. H. Ward, P. G. Hallof, W. R. Sill, and P. H. Nelson, 1978 Mineral discrimination and removal of inductive coupling with multifrequency IP: Geophysics, 43, 588-609, doi: 10.1190/1.1440839.

Pollock, D., and O. A. Cirpka, 2012, Fully coupled hydrogeophysical inversion of a laboratory salt tracer experiment monitored by electrical resistivity tomography: Water Resources Research, 48, W01505, doi: 10.1029/2011WR010779.

Revil, A., 2012, Spectral induced polarization of shaly sands: Influence of the electrical double layer: Water Resources Research, 48, W02517, doi: 10.1029/2011WR011260.

Revil, A., 2013. Effective conductivity and permittivity of unsaturated porous materials in the frequency range $1 \mathrm{mHz}-1 \mathrm{GHz}$ : Water Resources Research, 49, 306-327, doi: 10.1029/2012WR012700.

Revil, A., G. Z. Abdel Aal, E. A. Atekwana, D. Mao, and N. Florsch, 2015a, Induced polarization response of porous media with metallic particles Part 2: Comparison with a broad database of experimental data: Geophysics, 80, no. 5, D539-D552, doi: 10.1190/geo2014-0578.1.

Revil, A., A. Binley, L. Mejus, and P. Kessouri, 2015b, Predicting permeability from the characteristic relaxation time and intrinsic formation factor of complex conductivity spectra: Water Resources Research, 51, 6672-6700, doi: 10.1002/2015WR017074.

Revil, A., A. Coperey, Z. Shao, N. Florsch, I. L. Fabricius, Y. Deng, J. R. Delsman, P. S. Pauw, M. Karaoulis, P. G. B. de Louw, E. S. van Baaren, W. Dabekaussen, A. Menkovic, and J. L. Gunnink, 2017a, Complex conductivity of soils: Water Resources Research, 53, 7121-7147, doi: 10 1002/wrcr.v53.8.

Revil, A., and N. Florsch, 2010, Determination of permeability from spectral induced polarization data in granular media: Geophysical Journal International, 181, 1480-1498, doi: 10.1111/j.1365-246X.2010.04573.x.

Revil, A., N. Florsch, and D. Mao, 2015c, Induced polarization response of porous media with metallic particles - Part 1: A theory for disseminated semiconductors: Geophysics, 80, no. 5, D525-D538, doi: 10.1190/ geo2014-0577.1.

Revil, A., M. Karaoulis, S. Srivastava, and S. Byrdina, 2013, Thermoelectric self-potential and resistivity data localize the burning front of underground coal fires. Geophysics, 78, no. 5, B259-B273, doi: 10.1190/ geo2013-0013.1.

Revil, A., D. Mao, Z. Shao, M. F. Sleevi, and D. Wang, 2017b, Induced polarization response of porous media with metallic particles - Part 6 : The case of metals and semi-metals: Geophysics, 82, no. 2, E97-E110, doi: 10.1190/geo2016-0389.1.

Revil, A., C. D. Meyer, and Q. Niu, 2016, A laboratory investigation of the thermoelectric effect: Geophysics, 81, no. 4, E243-E257, doi: 10.1190/ geo2015-0281.1.

Revil, A., and M. Skold, 2011, Salinity dependence of spectral induced polarization in sands and sandstones: Geophysical Journal International, 187, 813-824, doi: 10.1111/j.1365-246X.2011.05181.x.

Revil, A., M. F. Sleevi, and D. Mao, 2017c, Induced polarization response of porous media with metallic particles - Part 5: Influence of the background polarization: Geophysics, 82, no. 2, E77-E96, doi: 10.1190/ geo2016-0388.1.

Rodriguez, B. D., 1983, A self-potential investigation of a coal mine fire: M.S. thesis, Colorado School of Mines.

Rosen, L. A., J. C. Baygents, and D. A. Saville, 1993, The interpretation of dielectric response measurements on colloidal dispersions using the dynamic Stern layer model: Journal of Chemical Physics, 98, 4183-4194 doi: $10.1063 / 1.465108$

Rosen, L. A., and D. A. Saville, 1991, Dielectrical spectroscopy of colloidal dispersions: Comparisons between experiment and theory: Langmuir, $\mathbf{7}$, 36-42, doi: 10.1021/la00049a009.

Rushworth, P., B. D. Haefner, J. L. Hynesand, and R. K. Streufert, 1989 , Reconnaissance study of coal fires in inactive Colorado coalmines: Colorado Geological Survey, Department of Natural Resources (Information Series 26, Denver Colorado, 60).

Saibaba, A. K., 2013, Fast algorithms for geostatistical inverse problems and uncertainity quantification: Ph.D. thesis, Stanford University. 
Saibaba, A. K., and P. K. Kitanidis, 2015, Fast computation of uncertainty quantification measures in the geostatistical approach to solve inverse problems: Advances in Water Resources, 82, 124-138, doi: 10.1016/j. advwatres.2015.04.012.

Schlumberger, C., 1920, Etude sur la prospection électrique du sous-sol: Ed. Gauthiers-Villars et Cie.

Seigel, H. O., 1949, Theoretical and experimental investigations into the applications of the phenomenon of overvoltage to geophysical prospecting: Doctoral dissertation, University of Toronto.

Seigel, H. O., 1959. Mathematical formulation and type curves for induced polarization: Geophysics, 24, 547-565, doi: 10.1190/1.1438625.

Sogade, J. A., F. Scira-Scappuzzo, Y. Vichabian, W. Shi, W. Rodi, D. P. Lesmes, and F. D. Morgan, 2006, Induced-polarization detection and mapping of contaminant plumes: Geophysics, 71, no. 3, B75-B84, doi: 10 $.1190 / 1.2196873$.

Soueid Ahmed, A., A. Jardani, A. Revil, and J. P. Dupont, 2014, Hydraulic conductivity field characterization from the joint inversion of hydraulic heads and self-potential data: Water Resources Research, 50, 35023522, doi: 10.1002/2013WR014645.

Soueid Ahmed, A., A. Jardani, A. Revil, and J. P. Dupont, 2015, HT2DINV: A 2D forward and inverse code for steady-state and transient hydraulic tomography problems: Computers \& Geosciences, 85, 36-44, doi: 10 .1016/j.cageo.2015.08.009.

Soueid Ahmed, A., A. Jardani, A. Revil, and J. P. Dupont, 2016, Specific storage and hydraulic conductivity tomography through the joint inversion of hydraulic heads and self-potential data: Advances in Water Resources, 89, 80-90, doi: 10.1016/j.advwatres.2016.01.006.

Spies, B. R., 1983, Recent developments in the use of surface electrical methods for oil and gas exploration in the Soviet Union: Geophysics, 48, 1102-1112, doi: 10.1190/1.1441532.
Van Voorhis, G. D., P. H. Nelson, and T. L. Drake, 1973, Complex resistivity spectra of porphyry copper mineralization: Geophysics, 38, 49-60, doi: 10.1190/1.1440333.

Vinegar, H., and M. Waxman, 1984, Induced polarization of shaly sands: Geophysics, 49, 1267-1287, doi: 10.1190/1.1441755.

Webster, R., and M. Oliver, 2001, Geostatistics for environmental scientists: Wiley \& Sons.

Wong, J., 1979, An electrochemical model of the induced polarization phenomenon in disseminated sulfide ores: Geophysics, 44, 1245-1265, doi: 10.1190/1.1441005.

Wong, A. S., N. D. Stalnaker, C. J. Laffer, J. D. Roberts, and J. H. Kuhr, 1996, The ion exchange properties of low rank coals on actinides and heavy metals: Abstract American Chemical Society, 481-483.

Xi, X. L., H. C. Yang, X. F. Zhao, H. C. Yao, J. T. Qiu, R. J. Shen, and R. J. Chen, 2014, Large-scale distributed 2D/3D FDIP system based on ZigBee network and GPS: Symposium on the Application of Geophysics to Engineering and Environmental Problems (SAGEEP 2014), EEGS, Expanded Abstracts, 130-139.

Yeh, T. C. J., S. Liu, R. J. Glass, K. Baker, J. R. Brainard, D. Alumbaugh, and D. LaBrecque, 2002, A geostatistically based inverse model for electrical resistivity surveys and its applications to vadose zone hydrology: Water Resources Research, 38, 14-1-14-13, doi: 10.1029/ 2001WR001204.

Zimmermann, E., J. Berwix, W. Glaas, H. Meier, H. M. Münch, and A Kemna, 2007, ZEL-SIP04-V02: User manual: Forschungszentrum Julich $\mathrm{GmbH}$.

Zimmermann, E., A. Kemna, J. Berwix, W. Glaas, H. Munch, and J. Huisman, 2008, A high-accuracy impedance spectrometer for measuring sediments with low polarizability: Measurement Science and Technology, 19, 105603, doi: 10.1088/0957-0233/19/10/105603. 